\title{
Fibrações de Milnor de singularidades analíticas reais
}

\author{
Raimundo Nonato Araújo dos Santos
}

Orientadora: Profa. Dra. Maria Aparecida Soares Ruas

Tese apresentada ao Instituto de Ciências Matemáticas e de Computação - ICMC-USP, como parte dos requisitos para obtenção do título de Doutor em Ciências - Matemática.

USP - São Carlos

Abril de 2002

Tiste traball

nciado pelo CNPq 


\section{A Comissão Julgadora:}

Profa. Dra. Maria. Iparecida Soares Ruas

Prof. I) Horco thenes Teixeira

Prof. Ir. Bruno (esar Azevedo Lcardua

Profa. Lra. léra Lúcia c carrara

Prof. Dr. Washington Luiz Marar

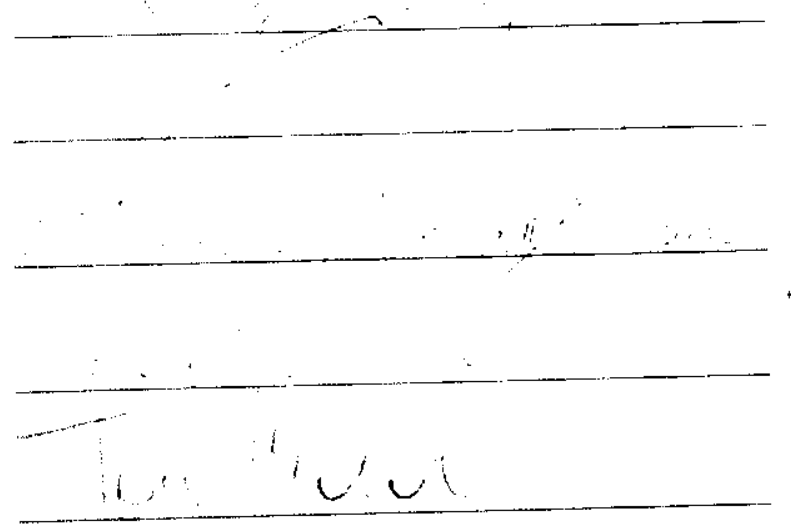


"A MEUS P AIS: Andremaria Santos e Antonio Carlos, in memoriam." 


\section{Agradecimentos}

Agradeço a Deus por mais uma conquista, e conseqüentemente, de todos os meus ascendentes.

A meus pais por todos os ensinamentos dia vida. in memoriam.

A professora e orientadora Maria Aparecida Soares Ruas (mainha) por sua infinita paciência, dedicação, e pelos ensinamentos na introdução da Teoria das Singularidades.

Aos amigos, Humberto, Liane e Alexandre, pelo empenho nas discussões e valorosas sngestões. Agradeço também ao amigo German pela ajuda com o Latiox.

Especialmente agradeço a duas pessoas fantást icas e fascinantes, com as qualis, se lous permitir, desejo dividir até o último momento de minha vida: Rosane o Nathaniel Minghim.

\section{Obrigado!!!}




\section{Resumo}

Neste trabalho estudamos a fibração de Milnor associada a singularidades isoladas reais definidas por germes de aplicacoios $f: \mathbb{R}^{n}, 0 \rightarrow \mathbb{R}^{2}, 0$. O principal resultado relaciona a existencia da fibraçào de Milnor com a (c)-regularidade la família de hipersuperfícies com singularidade isolada obtida projetando $f$ sobre a família $L_{-\theta}$ de todas as retas pela origem no plano $\mathbb{R}^{2}$. Fstudamos também famílias de germes de funçōes analíticas com singularidades isoladas. () objetivo é encontrar condiçóes suficientes para a trivialidade topológica das famílias e a equivalência das fibraçoes de Milnor associadas a elas. 


\section{Abstract}

In this work we study the Milnor's fibrations associated to real isolated singularities defined by map-germs $f: \mathbb{R}^{n}, 0 \rightarrow \mathbb{R}^{2}, 0$. The main result relates the existence of the Milnor's fibration with the (c)-regularity of the family of hypersurfaces with isolated singularity obtained by projecting $f$ into the family $L_{-} 0$ of all lines through the origin in the plane $\mathbb{R}^{2}$. We also study families of germs of andytic functions with isolated singularities. The aim is to get sufficient condition for the topological triviality of the families and the ecuivalence of the Milnor fibrations associated to them. 


\section{Índice}

Introdução

1 Preliminares 5

1.1 Fecho Integral . . . . . . . . . . . . . . . . . 6

1.2 Diagrama de Newton .................... 7

2 Fibraçāo de Milnor 11

2.1 A Condiçào do Milnor . . . . . . . . . . . . . . . . 12

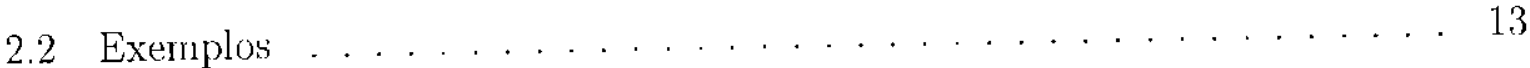

3 Fibração de Milnor Forte 16

3.1 Condições de Jacquemard . . . . . . . . . . . . . . . . 17

4 Principais Resultados 22

4.1 Condiçón de Rogularidade . . . . . . . . . . . . . . . . . 24

4.2 Resultado Principal para Fibraçóes de Milnor Reais . . . . . . . . . . . 26

4.3 Relação com os Resultados de Jacquemard . . . . . . . . . . . . . . 30

5 Famílias Analíticas Reais 35

5.1 Trivialização da Fibração de Milnor para Famílias Analíticas Reais . . . . . 37

5.2 Observaçóes finais . . . . . . . . . . . . . . . . . . . 45

Referências Bibliográficas $\quad 47$ 


\section{Introdução}

No final da década de 60, mais precisamente em 1968, John Milnor lançou seu livro "Singular Points of Complex Hypersurfaces", no qual estuda a topologia de uma varicdade analítica muma vizinhanç de um ponto crítico. Ele demonstra dois teoremas de fibração, um para germes de funçòes holomorfas e o outro para germes de aplicações arlalíticas reais.

No caso complexo, Milnor mostra que dado um germe de função holomorfa $\psi: \mathbb{C}^{n}, 0 \rightarrow \mathbb{C}, 0$, com singularidade isolada na origem, existe um $\epsilon_{0}>0$, suficientemente pequeno, tal que, para todo $0<t \leq f_{0}$, a aplicação

$$
\varphi:-\frac{\psi^{\prime}}{\|\psi\|}: S_{t}^{2 n-1} \backslash \Lambda_{t} \rightarrow S^{1}
$$

é a projeção de um fibrado localmente trivial, onde $K_{t}:=u^{-1}(0) \cap S_{\epsilon}^{2 n-1}$, é o link da singularidade.

No caso real, dizemos que un germe de aplicaçào polinomial $\psi: \mathbb{R}^{n}, 0 \rightarrow \mathbb{R}^{p}, 0, n \geq$ $p \geq 2$, satisfaz à condição de Milnor se cxiste uma vizinhança $U \subset \mathbb{R}^{n}$ da origem, tal que $f$ tem posto máximo $\mathrm{cm} U \backslash\{0\}$. Nilnor mostrou que existe um $c_{0}>0$, suficientemente pequeno, tal que para todo $0<c \leq \epsilon_{0}$, a aplicação $\varphi:=\frac{\psi}{\left|\psi^{\prime}\right|}: S_{\epsilon}^{n-1} \backslash N_{K_{\mathrm{r}}} \rightarrow S^{p}$ é a projeçäo de um fibrado localmente trivial, onde $N_{K_{\epsilon}}$ é uma vizinhança tubular de $K_{\epsilon}:=\psi^{-1}(0) \cap S_{t}^{n-1}$ na esfera $S_{t}^{n-1}$.

Milnor observa que sempre podemos provar que o complementar inteiro $S_{c}^{n-1} \backslash K_{\text {, }}$ também fibra sobre $S^{p-1}$, mas não é verdade em geral que a aplicaçâo projeção ainda permanece do tipo $x \mapsto \frac{\psi(x)}{\mid \cdot(x) \|}$. Como exemplo, exibe a seguinte aplicação: 


$$
\left\{\begin{array}{l}
P=x \\
Q=x^{2}+y\left(x^{2}+y^{2}\right)
\end{array}\right.
$$

Quando podemos estender o fibrado à $S_{\epsilon}^{n-1} \backslash K_{\epsilon}$, mantendo a aplicação $\frac{\psi^{\prime}}{|i|^{\prime}}$ como projeção do fibrado, dizemos que a aplicação é do tipo Milnor Forte.

Essa condição Milnor Forte foi estudada pela primeira vez por A. Jacquemard, no final da década de 80, no artigo "Fibrations de Milnor pour des applications réelles" [Ja]. Ele propõe duas condições suficientes, uma geométrica e outra algébrica, para que um germe de aplicação analítica $\psi: \mathbb{R}^{n}, 0 \rightarrow \mathbb{R}^{2}, 0$ tenha uma fibração deste tipo. Um problema, na prática. é verificar quando um dado germe satisfaz suas hipóteses, já que ele cxibe poucos excmplos de aplicaçóes que as satisfazem.

Em [[S1], [S2]], Scade dá uma construção quo fornece famílias infinitas de singularidacles isoladas reais $\psi: \mathbb{C}^{n}: 0 \rightarrow \mathbb{C}, 0$ satisfazendo à condição Milnor forte na origem, da seguinte forma: dado um germe em $0 \in U \subset \mathbb{C}^{n}$ de campo de vetores holomorfos $F$ e $X$, a funçào $\psi_{F, X}: U \subset \mathbb{C}^{n} \rightarrow \mathbb{C} \simeq \mathbb{R}^{2}$, definida por $\psi_{F, X}(z)=\langle F(z), X(z)\rangle=$ $\sum_{i=1}^{n} F_{i}(z) . \overline{X_{i}(z)}$, é analítica real. o podemos perguntar en que condiçôes esta aplicação tem singularidade isolada e satisfą à condição acima na origem. Uma classificação completa de tais exemplos quando $F$ ' $X$ são campos de vetores monomialis é dada cm [RSV].

Neste trabalho usamos o método de Seade para dar uma condição suficiente para que um germe de aplicação analítica real $\psi: \mathbb{R}^{n}, 0 \rightarrow \mathbb{R}^{2}, 0$, com singularidade isolada em 0 satisfaça à condição Milnor forte. Seja $\pi_{\theta}: \mathbb{C} \rightarrow L_{-\theta}$ a projeção linear sobre a reta $L \ldots_{\theta}$, formando um ângulo - $\theta$ com o cixo horizontal em $\mathbb{C} \simeq \mathbb{R}^{2}$. Nosso principal resultado estabelece que se a família $\psi_{0}=\pi_{\theta} \circ \%$ satisfaz à condição de $(c)$-regularidade de K. Bekka, então $\psi$ satisfaz à condição Milnor forte. Como unla consecuênencia deste resultado podemos provar que as hipóteses de Jacquemard são equivalentes a (w)-regularidade da família $\psi_{0}$. Estudamos também famílias de germes de aplicaçoes analíticas com singularidades isoladas. O objetivo é obter condiçòes suficientes para a trivialidade topológica destas fanílias e a equivalência das fibraçoes de Milnor a elas associadas.

No primeiro capítulo deste trabalho revemos fatos básicos sobre diagrama de Newton e fecho integral de ideais, e cmmeiamos um resultado de M.Saia [Ma], que relaciona o fecho integral de um ideal Newton não-degenerado com o ideal gerado por monômios cujos expoentes aparecem nas faces do diagrana de Newton.

No capítulo 2, relembramos os teoremas de fibraça de Milnor [Mi], e mostramos 
que para germes analíticos a condição de Milnor é determinada por um jato de ordem finita. Enunciamos um resultado devido a Ruas, Seade e Verjovski, dado em [RSV], para fibraçóces do tipo quase-homogêneas, o qual fornece ordens precisas para perturbaçốs que preservam a condição de Milnor. Finalizamos com uma caracterização do singularidade do tipo Milnor em $\mathbb{C}$, e mostramos como reobter a partir deste exemplo, uma das famílias classificadas em [RSV].

No capítulo 3, apresentamos o resultado de A. Jaccuemard e provamos um primeiro resultado sobre a condiçào Milnor forte para germes cujos gradientes das funçóes coordenadas são quase-homogêneos.

O capítulo 4, contém os principais resultados do trabalho. No Teorma 4.2.2, relacionamos a existência da fibração de . Vilnor com a (c)-regularidade da família $\psi_{\theta}$ de hipersuperfícies com singularidade isolada, obtida por projeções sobre a famílid de retas no plano passando pela origem. Mostramos ainda que a $(w)$-regularidade desta família é condição equivalente às condiçoes de Jaçuemard. Discutimos exemplos que mostram que a classe das singularidades que satisfazem à condição . Vilnor forte contém propriamente a classe das singularidades cujas famílias $\psi_{0}$ associadas satisfazem à condição de (c)-regularidade; a qual, por sua vez, contém propriamente aquelas que satisfazem às condiços $(A)$ e $(B)$ de Jacquemard.

No último capítulo, o capítulo 5 , verificamos como se comportam as fibrações do tipo Milnor forte, ao longo de famílias de aplicaçoes analíticas reais com singularidade isoladd. Mostramos que as fanílias que satisfazen às hipóteses de Jaccuemard são topologicamente triviais e as fibraçoes de Milnor associadas são equivalentes. Além disso, para germes cujas funções coordenadas possuem gradientes Newton não-degenerados, mostramos que a condição Milnor forte e o tipo topológico da fibração permanecem invariantes por pertubações por termos de filtraça maior ou igual à filtração de Newton do germe inicial. 


\section{Capítulo 1}

\section{Preliminares}

Vamos introduzir neste capítulo as notações e definiçōes básicas, usualmente utilizadas na Teoria de Singularidades.

Seja $\mathcal{A}_{n}=\left\{f: \mathbb{R}^{n}, 0 \rightarrow \mathbb{R}, 0\right\}$, o conjunto dos gemes na origem de funçoes analíticas reais. É conhecido que $\mathcal{A}_{n}$ é um anel local cujo ideal maximal denotamos $\mathcal{M}_{n}$.

Mais geralmente, $\mathcal{A}(n, p)=\left\{f: \mathbb{R}^{n}: 0 \rightarrow \mathbb{R}^{p}, 0\right\}$, onde $f$ ć uma aplicação analítica real, é um módulo sobre o ancl $\mathcal{A}_{n}$. Em particular, quando $p=1$, temos $\mathcal{A}(n, 1)=\mathcal{A}_{n}$.

Indicaremos por $\mathcal{O}_{n}=\left\{f: \mathbb{C}^{\prime \prime}, 0 \rightarrow \mathbb{C}, 0\right\}$, o anel dos germes na origom de funções holomorfas.

Neste trabalho estaremos especialmente interessados no módulo $\mathcal{A}(n, p)$, quando $p=2$. Todo elemento $f \in \mathcal{O}_{n}$ pode ser considerado como um clemento em $\mathcal{A}(n, 2)$.

Considere $\mathcal{C}^{l}-\mathcal{R}=\left\{h: \mathbb{R}^{n}, 0 \rightarrow \mathbb{R}^{n}, 0\right\}$, onde $h$ é un difomorfismo de classe $\mathcal{C}^{l}$, $0 \leq l \leq \infty$. Com a operação de composição, $\mathcal{C}^{l}-\mathcal{R}$ é um grupo, também conhecido como o grupo de mudança de coordenaclas $\mathcal{C}^{l}$ na fonte.

Para cada $0 \leq l \leq \infty$, existe una ação natural do grupo $\mathcal{C}^{l}-\mathcal{R}$ à direita no espaço dos germes de classe $\mathcal{C}^{l}$, mas extaremos interessados na restriçâa desta ação ao módulo $\mathcal{A}(n, p)$.

Definição 1.0.1 Sejam $f, g: \mathbb{R}^{n}, 0 \rightarrow \mathbb{R}^{p}, 0$ germes de aplicaçöes analíticas. Dizemos que f e g são $\mathcal{C}^{l}-\mathcal{R}$-equivalentes, e denotamos $f_{\mathcal{C}^{-}-\mathcal{R}}$ g. se existe um germe de difeomorfismo de classe $\mathcal{C}^{l}, l \geq 0, h: \mathbb{R}^{n}, 0 \rightarrow \mathbb{R}^{n}, 0$, tal que $g=f \circ h$.

Definição 1.0.2 Um elemento $f \in \mathcal{A}(n, p) e^{\prime} k-\mathcal{C}^{l}-\mathcal{R}$ - determinado se $\forall g \in \mathcal{A}(n, p)$ 
tal que $j^{k} f(0)=j^{k} g(0)$, enlão $f \underset{0^{?}-R}{\sim} g$. Dizemos que $f$ é finitamente determinado se $f$ é $k$-determinado para algum $k$.

Dada $f: \mathbb{R}^{n}, 0 \rightarrow \mathbb{R}^{p} ; 0$ denotanos por $\mathrm{I}_{\mathcal{R}} f$ o ideal de $\mathcal{A}_{n}$ gerado pelos menores $p \times p$ da matriz jacobiana de $f$, e por $N_{R} f(x)=|d f(x)|^{2}=\sum_{j} \mathrm{M}_{j}^{2}$, onde os $\mathrm{M}_{j}$ são os geradores de $\mathrm{I}_{R} f$.

Definição 1.0.3 Dizemos que f satisfaz a uma condição de Lojasiewicz, se existem constantes positivas c e $\alpha$ lais que $\|f(x)\| \geq c|| x \|^{\alpha}$, numa vizinhança da origem.

A seguinte proposição resulta do trabalho de vários autores ([K], [Ku], [BL]). Veja [W] para um survey sobre o assunto.

Proposição 1.0.1 Seja $f \in \mathcal{A}(n, p)$. Säo equivulentes:

(a) $\sum_{f}=\{0\}$, onde $\sum_{f}$ denota o germe do conjunto singular de $f$.

(b) $\mathrm{N}_{\mathcal{R}} f(x)$ satisfaz a uma condição de Lojasiewicz;

(c) $f$ é $\mathcal{C}^{l}-\mathcal{R}$-finitamente determinado para todo $l \in[0, \infty)$.

Para estimativas para o grau de $\mathcal{C}^{l}$-determinação finita, veja [RS], [R].

\section{$1.1 \quad$ Fecho Integral}

Vamos relembrar a noção de fecho integral de um ideal. As principais referências para esta seção sâo [Te], [Ma], [CR].

Definição 1.1.1 Seja I um ideal do anel $A$, entûo $h \in A$ está no fecho integral de $I$, denotado por $\bar{I}$, se existe um polinômio mônico $P(z)=z^{n}+\sum_{i} a_{i} z^{i}, a_{i} \in I^{n-i}$, tal que $P(h)=0$.

Quando o ancl $A=\mathcal{O}_{u}$ : Teissier [Te] exibiu várias noşões que são equivalentes:

Teorema 1.1.1 ([Te]) Seja I um ideal em $\mathcal{O}_{n}$. Sào equivalentes: 
Definição 1.2.2 O diagrama (ou poliedro) de Newton de $I$, denotado por $\Gamma_{+}(I)$, fo fecho convexo em $\mathbb{R}_{+}^{n}$ do conjunto $E(I)$. Denotaremos por $\Gamma(I)$ a uniäo das faces compactas de $\Gamma_{+}(I)$.

Considere $I=\left\langle g_{1}, \ldots, g_{s}\right\rangle$ um ideal finitamente gerado e de codimensão finita em $\mathcal{O}_{n}$.

Dado um vetor $v \in \mathbb{R}_{+}^{n} \backslash\{0\}$, definimos $l(v)=\min \left\{\langle k, v\rangle: k \in \mathrm{I}_{+}{ }_{+}(I)\right\}$.

Uma definição formal para a face de um poliedro é a seguinte: um subconjunto $\Delta$ de $\Gamma_{+}(I)$ é uma face quando existe $v \in \mathbb{R}_{+}^{n} \backslash\{0\}$ tal que $\Delta=\left\{k \in \Gamma_{+}(I):\langle k, v\rangle=l(v)\right\}$. Nesse caso, $v$ define a face $\Delta$. Quando $v$ co o vetor de menor comprimento que define $\Delta e$ pertence ao conjunto $\left(\mathbb{R}_{+}^{n} \backslash\{0\}\right) \cap \mathbb{Z}_{1}^{n}$, v ć chamado um vetor primitivo para $\Delta$.

Dado um subconjunto finito $\Delta \subset \Gamma_{+}(I)$, para qualquer germe $f(x)=\sum a_{i} x^{i}$, definimos $f_{\Delta}=\Sigma_{k \in \Delta} a_{k} x^{k}$. Se $\Delta$ é uma face de $\Gamma_{+}(I), C(\Delta)$ denota o cone formado pelos semi-raios saindo da origem e passando por $\Delta$.

Como $I$ é um ideal de codimensão finita em $\mathcal{O}_{n}$ e $\Gamma_{-}(I)$ é um poliedro convexo em $\mathbb{R}_{+}^{n}$, a coleção de todos $C(\Delta)$ dá uma decomposição poliedral para $\mathbb{R}_{+}^{n}$.

Antes de construirmos a filıração de Newton em $\mathcal{O}_{n}$, vamos lembrar da filtração homogênca. Dado urn germe $f(x)=\sum a_{k} x^{k} \in \mathcal{O}_{n}$, cscrevemos $f=f_{d}+f_{d+1}+\ldots$ em que $f_{i}$ é uma forma de grau $i$, isto é, um polinómio homogêneo de grau $i$. O grau de homogeneidade é medido pela valoração $v_{h}: \mathcal{O}_{n} \rightarrow \mathbb{R} \cup\{\infty\}, v_{h}(f)=\operatorname{nin}\{\varphi(k): k \in \operatorname{supp}(f)\}$ em que $\varphi: \mathbb{R}_{+}^{n} \rightarrow \mathbb{R} \cup\{\infty\}, \varphi(k)=\varphi\left(k_{1}, \ldots, k_{n}\right)=k_{1}+\ldots+k_{n}$. O conjunto $\varphi^{-1}(d)$, que denotamos por $\Delta_{\text {, }}$ é face de nível d da filtração homogênea. Observamos que a função $\varphi$ satisfaz as seguintes propriedades:

(1) $\varphi$ ó linear em $C\left(\Delta_{1}\right)$

(2) $\varphi$ assume valores inteiros positivos em $\mathbb{Z}_{+}^{n} \bigcap \mathbb{R}_{+}^{n}$

(3) $\varphi$ é constante na face compacta $\Delta_{1}$

Para generalizarmos esta idéia, fixamos um poliedro $\Gamma_{+}(I)$ e definimos a seguinte aplicação

$$
\Phi: \mathbb{R}_{+}^{n} \rightarrow \mathbb{R} \cup\{\propto\}
$$

satisfazendo: 
(1) $h \in \bar{I}$.

(2) (Condição de Crescimento) Para cada escolha de geradores $g_{i}$ de I existem uma vizinhança $U$ de 0 e uma constante $C>0$ tal que para todo $x \in U$ :

$|h(x)| \leq C \cdot \sup _{i}\left|g_{i}(x)\right|$

(3) (Critério Avaliativo) Para cada curva analítica $\varphi:(\mathbb{C}, 0) \rightarrow\left(\mathbb{C}^{n}, 0\right)$, $h \circ \varphi \in\left(\varphi^{*}(I)\right) \mathcal{O}_{1}$.

(4) Existe um módulo fiel $M$ sobre $\mathcal{O}_{n}$ tal que h.M $M \subset I . M$.

A definição algébrica de fecho integral não ć a mais adcquada para o estudo de germes analíticos reais, como observou Gaffney em [Ga]. Por exemplo, considerando o ideal $I=\left(x^{2}+y^{2}\right) \mathrm{em} \mathcal{A}_{2}$, esperamos pela condição (3) e (4) acima, que o fecho integral real seja $\mathcal{M}_{2}^{2}=\left(x^{2}, x y, y^{2}\right)$, mas verifica-se que $\mathcal{M}_{2}^{2}$ contém propriamente $\bar{I}$.

Como estaremos interessados en germes de aplicaços analíticas reais, vamos introduzir a definição de fecho integral real dada por Gaffney em $\left.{ }_{i} \mathrm{Ga}\right]$.

Definição 1.1.2 Dado $h \in \mathcal{A}_{n}, h \in \bar{I}_{\mathrm{*}}$, e lê-se $h$ está no fecho integral real de $I$, se para qualquer curva analitica real $\varphi:(\mathbb{R}, 0) \rightarrow\left(\mathbb{R}^{n}, 0\right)$, temos que

$$
\nu(h \circ \varphi) \geq \min _{i} \nu\left(g_{i} \circ \varphi\right) ; I-\left(g_{1}, \ldots, g_{s}\right)
$$

onde $\nu$ a valoraçäo canônica no anel das séries de potências convergentes na origem.

Observamos que a definição acina é equivalente a dizer que para toda curva analítica real, $\varphi:(\mathbb{R}, 0) \rightarrow\left(\mathbb{R}^{n}, 0\right)$ temos $h \circ \varphi \in\left(\varphi^{*}(I)\right) \mathcal{A}_{1}$. Gaffney prova em [Ga] que a equivalència $(2) \Longleftrightarrow(3)$ ainda ó verdadeira para ideais sm $\mathcal{A}_{n}$

\subsection{Diagrama de Newton}

Dado un germe $f(x)=\Sigma a_{i} x^{i}$ em $\mathbb{R}^{n}$, o suporte de $f$ c definido por supp $(f)=\{j \in$ $\left.\mathbb{Z}^{n} ; a_{j} \neq 0\right\}$.

Definiçāo 1.2.1 Seja I um idcul de $\mathcal{O}_{n}$. Definimos supp $(I)=\bigcup\{\operatorname{supp} f: f \in I\}$ e, $E(I)=\bigcup\left\{k+v: k_{i} \in \operatorname{supp}(I) . v \in \mathbb{R}_{+}^{n}\right\}$. 
(1) para cada face $\Delta$ de $\Gamma_{+}(I)$, $\Phi$ é linear em $C(\Delta)$,

(2) $\Phi$ assume valores inteiros positivos em $\mathbb{Z}_{+}^{n} \bigcap \mathbb{R}_{+}^{n}$.

(3) existe $p \in \mathbb{N}$ tal que $\left.\Phi\right|_{\Gamma^{\prime}(I)}=p$.

Definimos $f l l: \mathcal{O}_{n} \rightarrow \mathbb{R} \cup\{\infty\} \operatorname{com} f u l(f)=\inf \{\Phi(k): k \in \operatorname{supp}(f)\}$. Como $\operatorname{supp}(0)=\varnothing$, convencionamos então $f i l(0)=\infty$.

Lema 1.2.1 fil é uma funçäo de ordem em $\mathcal{O}_{n}$.

$\Lambda$ demonstração do Loma acima, é de fácil verificagão, mas para una prova direta e fácil, assim como una melhor apresentação sobre o assunto, convido o leitor a consultar [CR].

Definimos para cada $\Delta \subset \Gamma(I)$, o anel $C[[\Delta]]$, consistindo das séries de potências com monômios nấo nulos $x^{s}=x_{1}^{s_{1}} \ldots x_{n}^{s_{n}}$ tais quo $s=\left(s_{1}, \ldots, s_{n}\right) \in C(\Delta)$.

Considere ajnda $I=\left(g_{1}, \ldots, g_{s}\right)$, finitamente gerado $\mathrm{cm} \mathcal{O}_{n}$.

Definição 1.2.3 Dado um ideal $I$ em $\mathcal{O}_{n}$, denotamos por $C(\bar{I})$ o fecho convexo em $\mathbb{R}_{+}^{n}$ do conjunto $\left\{k \in \mathbb{N} \mid x^{k} \in \bar{I}\right\}$

Definição 1.2.4 Uma face compacta $\Delta \subset \Gamma(I)$ é Neuton năo-degenerada se o ideal gerado por $g_{1_{\Delta}}, \ldots, g_{n_{\Delta}}$ tem codimensáo finita em $C[[\Delta]]$.

Definição 1.2.5 Um ideal I é Neuton nüo-degenerado se cada face compacta $\Delta \subset \Gamma(I)$ é Newton näo-degenerada.

Observamos que a definiçào acima é equivalente do seguinte:

$I$ é Newton não-degenerado se para carla face compacta $\Delta \subset \Gamma(I)$, a equação $g_{1_{\Delta}}=\ldots=g_{n_{\Delta}}=0$ não tem solusăo comum em $(\mathbb{C}-\{0\})^{n}$.

O principal resultado, devido a Marcelo Saia [Ma], que usaremos neste trabalho caracteriza os ideais Newton não-degenerados $\mathrm{m}$ função do fecho integral, para os ideais finitamente gerados e do codimensào finita em $\mathcal{O}_{n}$.

Teorema 1.2.2 ([Ma $])$ Seja $I=\left\langle g_{1}, \ldots, g_{s}\right\rangle$ um ideul de rodimensão finita em $\mathcal{O}_{n}$. Então, I é. Neuton não-degenerado se e somente se, $\mathrm{J}_{+}(I)=C(\bar{I})$. 
Observação: Os conceitos desta sção se estendem para germes e ideais em $\mathcal{A}_{n}$. O Teorema 1.2 .2 também permanece verdadeiro em $\mathcal{A}_{n}$. Isto é, se $I \subset \mathcal{A}_{n}$ é um ideal de codimensão finita Newton não-degenerado, então $\Gamma_{-}(I)=C(\bar{I})$ ([Mal]). 
CAPÍtulo 2

\section{Fibração de Milnor}

Seja $f:\left(\mathbb{C}^{n+1}, 0\right) \rightarrow(\mathbb{C}, 0)$ um germe de funçăo holomorfia com singularidade isolada. Considere $V:=f^{-1}(0) . S_{f}:=\left\{z \in \mathbb{C}^{n} /|z|-\epsilon\right\}$ e $K_{f}:=f^{1}(0) \cap S_{\text {( }}$ (link da singularidade). Em seu livro "Singular Points of Complex Iypersurfaces", 1968, John Milnor provou o seguinte:

Teorema 2.0.3 Existe $\epsilon_{0}>0$, suficientemente pequeno, tal que $\varphi=\frac{f}{\|f\|}: S_{\epsilon}^{2 n+1} \backslash K_{\epsilon} \rightarrow S^{1}$ é a projeção de um fibrado sunve localmente trivial.

Slém disso, a fibra $F_{\theta}:=\varphi^{-1}(\exp (i \theta))$ é uma $2 n$-subvariedade real suave de $S_{t}$, paralelizável e que tem o tipo de homotopia de um bouquet com $\mu(f)$ esferas $S^{n}$, onde $\mu(f)$ é o núnero de Vilnor da singularidade, e a subvariedade $K_{c}$ é $(n-2)$-conexa.

Para o caso de singularidades reais, Milnor também provou um teorema de fibração que passamos a descrever:

Seja $f:\left(\mathbb{R}^{m}, 0\right) \rightarrow\left(\mathbb{R}^{k}, 0\right)$ una aplicação polinomial com $f(0)=0$ tal que existo uma vizinhança $U \subset \mathbb{R}^{m}, 0 \in U$ com $\operatorname{rank}(J f(x))=k, \forall x \in U \backslash\{0\}$. Ou seja, $f$ tem posto máximo numat vizinhança da origen, exceto possivelnente rm 0 .

Sabemos que, noste caso, $V:=\left\{x \in \mathbb{R}^{m} / f_{1}(x)=\ldots=f_{k}(x)=0\right\}$ é uma variedade suave de dimensão in-k em $(U \cap V) \backslash\{0\}$ ou é vazio. Alćm disso a interseção $K_{\epsilon}:=V \cap S_{\epsilon}^{m-1}$ c uma variedade suave de dimensão $=m-\mathrm{k}-1$, para $\epsilon>0$ suficientemente pequeno.

Vamos supor de agora em diante que $k \geq 2$. O Teorema principal de Milnor neste caso é:

Teorema 2.0.4 O complementar de uma vizinhança tubular aberta de $K_{c}$ em $S_{\epsilon}^{m-1} e ́$ 
o espaco total de um fibrado suave sobre a esfera $S^{k}{ }^{1}$, cada fibra $F$ sendo uma ( $m$-k)variedade compacta suave cujo bordo é uma cópia de $K_{\epsilon}$.

As demonstraçoes dos teoremas no caso real e complexo podem ser encontradas em [Mi]. Neste capítulo, apresentamos a teoria e algunas ferramentas básicas para o estudo de fibraçōes de Milnor Reajs.

\subsection{A Condição de Milnor}

Vamos agora introduzir algumas dofiniçoes e resultados yue nos servirão para os próximos capítulos. As principais referências são [RS], [RSV! e [W].

Definição 2.1.1 Dizemos que uma aplicaçao analituca $f:\left(\mathbb{R}^{m}, 0\right) \rightarrow\left(\mathbb{R}^{k}, 0\right)$ satisfaz à condiçäo ou hipótese de Milnor. ou é de Milnor, se fxiste uma vizinhança die origem $U \subset \mathbb{R}^{m}$ tal que rank $(J f(x))=k$ para todo $x \in U \backslash\{0\}$.

Do ponto de vista da Teoria de Singularidades esta propriedade pode ser bem caracterizada através do conceito do $\mathcal{C}^{l}-\mathcal{R}$-equivalência, pela Definição 1.0 .2 do capítulo 1.

A seguinte caracterização para a hipótese de Milnor é uma consecquência imediata da Proposiçāo 1.0.1.

Teorema 2.1.1 Seja $f \in \mathcal{A}(m, p)$. Sào cquivalcntes:

(a) f satisfaz a uma condiçäo de Milnor em 0;

(b) $\mathrm{N}_{\mathbb{R}} f(x)$ satisfaz a uma condição de Lojasiewicz;

(c) fé $\mathcal{C}^{l}-\mathcal{R}$-finilumente determinado para todol $l \in[0, \infty)$.

Quando $f$ é una aplisaçào quase lomogênea, cxistem estimativas precisas para a ordem das perturbaçós que preservam a condição de Milnor. Vejamos antes algumas definições e notações.

Definição 2.1.2 Dades $r_{1}, \ldots, r_{n} ; d_{1}, \ldots, d_{p}$. inteiros positivos, um elemento $f \in \mathcal{A}(n, p) e ́$ quase homogêneo do tipo $\left(r_{1}, \ldots, r_{n}: d_{1}, \ldots, d_{p}\right)$ se para lodo $\lambda>0$ temos: $f\left(\lambda^{r_{1}} x_{1}, \ldots, \lambda^{r_{n}} x_{n}\right)=$ 
$\left(\lambda^{d_{1}} f(x), \ldots, \lambda^{d_{n}} f(x)\right)$. Os números $r_{1}, \ldots, r_{n}$ são os pesos de $x_{1}, \ldots, x_{n}$. O inteiro $d=$ $d_{1}+\ldots+d_{n}$. co peso total de $f$. Observamos que esses inteiros não săo únicos.

Definição 2.1.3 (i) Dados posos $\left(r_{1}, \ldots, r_{n}\right)$ para $\left(x_{1}, \ldots, x_{n}\right)$, e um monômio $x^{n}=$ $x_{1}^{\alpha_{1}} \ldots x_{n}^{\alpha_{n}}, \alpha=\alpha_{3}+\ldots+\alpha_{n}$, definimos sua filtraçäo por: full $\left(x^{(\kappa)}\right)=\sum \alpha_{i} x_{i}$.

(ii) Dado $f \in \mathcal{A}_{n}$, sua fillracào é: $f i l(f)=\inf _{\alpha}\left\{f i l\left(x^{(k)}\right) /\left(\frac{\partial^{\alpha} f}{\partial x^{\alpha}}\right)(0) \neq 0\right\}$, onde $\frac{\partial^{\alpha} f}{\partial x^{a}}$ significa a derivada parcial de $f$ com respeito aos $x_{t^{\prime} s}$, com grau total $\alpha$.

(iii) Dado $f=\left(f_{1}, \ldots f_{p}\right) \in \mathcal{A}(n, p)$, com fill $\left(f_{i}\right)=d_{i}$, a filtracüo de $f$ é fil $(f)=$ $\left(d_{1}, \ldots, d_{p}\right)$

Neste caso temos:

Teorema 2.1.2 ([RS], Teorema 2.2) Seja $f: \mathbb{R}^{m}, 0 \rightarrow \mathbb{R}^{p}, 0$ germe de aplicaçăo polinomial quase homogêneo, do tipo $\left(r_{1}, \ldots, r_{m} ; d_{1}, \ldots, d_{p}\right)$ com $r_{1} \leq \ldots \leq r_{m}, d_{1} \leq \ldots \leq d_{p}$, com singularidade isolada. Entio:

(a) Deformaçies de f definidas por $f_{t}(x)=f(x)+t \theta(x), \theta(x)=\left(\theta_{1}, \ldots, \theta_{p}\right)$ com fil $\left(\theta_{i}\right) \geq$ $d_{i}-r_{1}+r_{m}+1$, para lodo $\dot{x}, l \geq 1$ ot $\in[0,1]$ säo $\mathcal{C}^{l}-\mathcal{R}$-triviais.

(b) Se fi é uma deformagäo de f qué quase homogenea do mésmo tipo que $f$ então a familia $f_{t} e^{0} \mathcal{C}^{0}-\mathcal{R}$ erivial parat suficientemente pequeno.

\subsection{Exemplos}

Exemplos de germes $f: \mathbb{R}^{2 m}, 0 \rightarrow \mathbb{R}^{2}, 0 \mathrm{com}$ singularidade isolada que nào provêm de um germe de aplicação holomorfa $f:\left(\mathbb{C}^{m}, 0\right) \rightarrow(\mathbb{C}, 0)$ com singularidade isolada são difíceis de serem encontrartos.

Em [S1] e [S2], Seade descrevo num método para construir tais cxemplos e, em [RSV] sào exibidas famílias de singularidades que satisfazem à condigão de Milnor.

Sejan $F$ e $X$ germes de campos de vetores analíticos complexos em $\left(\mathbb{C}^{n}, 0\right)$. Defina a aplicação analítica real $\psi_{F, X} \in \mathcal{A}(2 n, 2)$ dada por $\xi_{F, X}:=\langle F, X\rangle$, onde $\langle$,$\rangle denota 0$ produto hermitiano $\mathrm{em} \mathbb{C}^{n}$. Assin,

$$
\varphi_{F, X}=\sum_{i} F_{i}(z) \overline{\bar{X}_{i}(z)}
$$


Como uma consequência do Teorema 2.1.2 temos:

Corolário 2.2.1 Supomha que o germe de aplicaçüo $\hat{r}_{r, x}$ satisfaça à condiçăo do Milnor em 0 . Sejam $F_{*}$ e $X_{*}$ campos de vetores analíticos, obtidos respectivamente, adicionando a $F$ e a X termos de grau suficientemente grande. Entăo a correspondenle funçäo $\psi_{F_{*}, x}$. sutisfaz à condiçăo de Milnor em 0. Além disso, exisle um germe de homeomorfismo

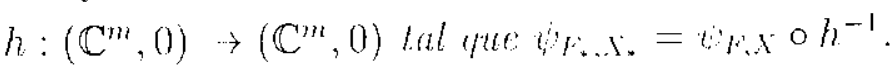

Sejam $F$ e $X$ campos monomiais $F(z)=\left(k_{1} z_{\sigma_{1}}^{a_{1}}, \ldots, k_{m} z_{\sigma_{n}}^{a_{m}}\right), X(z)=\left(t_{1} z_{1}^{b_{1}}, \ldots, l_{m} z_{m}^{b_{m}}\right)$, onde os $k_{i^{\prime} s}$ e $t_{i^{\prime} s}$ sào números complexos não mulos, $a_{i^{\prime} s}, b_{i^{\prime} s}$ são naturais e $\sigma=\left(\sigma_{1}, \ldots, \sigma_{m}\right)$ ó alguma permutação das coordenadas $\left(z_{1}, \ldots, z_{m}\right)$, cm [RSV] é obtida uma classificação completa das $\psi_{F_{2}, X}$ que satisfazem à condição de Milnor.

Teorema 2.2.2 ([RSV], Teorema 2.2) Suponha $a_{i}>1, \forall i=1, \ldots$, m. Fnläo satisfaz à condiçä de Milnot om $0 \in \mathbb{C}^{m}$ se, e somente se uma das seguintes condiçöes é satisfeita:

(a) A permulação $\sigma$ é a identidade. ou seja, $F(z)=\left(k_{1} \hat{y}_{1}^{a_{1}}, \ldots, k_{m} z_{m}^{a_{n}}\right)$ e $a_{i} \neq b_{i}$, para todo i.

(b) Se $\sigma$ se fatora em riclos $\sigma_{1}, \ldots, \sigma_{s}$, tais que cada $\sigma_{j}$ é uma permutação de comprimento $\theta_{j} \operatorname{com} \theta_{1}+\ldots+\theta_{s}=m$, então para cada $j=1, \ldots, s$ tal que $\theta_{j}=1$, devernos ter que o correspondente expoente satisfaz $a_{j} \neq b_{j}$; para cada $j=1, \ldots$, s com $\theta_{j}>1$, o expoente bj correspondente a csisas componentes säo todos 1 (ou 0$)$.

Vamos analisar no próximo exemplo en que condiçoes un campo $F:(\mathbb{C}, 0) \rightarrow(\mathbb{C}, 0)$ dá origem a unà aplicaçăo tr: $(\mathbb{C}, 0) \rightarrow(\mathbb{C}, 0), \varphi(z)=\langle F(z): X(z)\rangle=F(z) . \overline{X(z)}$, que satisfaz à condiçán de Milnor. É um exemplo simpless, mas como consequência desta análisc reobtemos o itcm (a) do Teorema 2.2 .2 acima. Além disso, podemos observar que a condição "Milnor" não ć aberta, isto ć, não permanece necessariamente verdadeira, por pequenas perturbaçös.

Exemplo 2.2.3 Seja $H^{\prime}:(\mathbb{C}, 0) \rightarrow(\mathbb{C} .0)$ holomorfa. Definamos $\psi(z)=F(z) \cdot \bar{X}(z)$, onde $X(z)=z$. Entiao:

(i) Se j $j^{1} F(0) \neq 0 \Rightarrow$ ir nù é de Milnor.

(ii) $S e \cdot j^{1} F(0)=0$ e $j^{n+1} F(0) \neq 0$. para algum $n \geq 1 \Rightarrow t e$ de Milnor. 
Vamos comecar com $F:\left(\mathbb{C},(0) \rightarrow(\mathbb{C}, 0), F(z)=\alpha z: \alpha \in \mathbb{C}^{*}, \log 0 ; \psi(z, \bar{z})=\right.$ $\langle F(z), z\rangle=\alpha z \bar{z}=\alpha|z|^{2}$. Temos,

$\psi_{1}=\operatorname{Re}(\psi)=(\alpha+\bar{\alpha})|z|^{2}$ e $\psi_{2}=\operatorname{lm}(\varphi)=(\alpha-\bar{\alpha})|z|^{2}$. Calculando a matriz jacobiana, obtemos:

$D \dot{\psi}=\left(\begin{array}{cc}(\alpha+\bar{\alpha}) \bar{z} & (\alpha+\bar{\alpha}) z \\ (\alpha-\bar{\alpha}) \bar{z} & (\alpha-\bar{\alpha}) z\end{array}\right) \therefore \operatorname{det} D \psi(z, \bar{z})-(\alpha+\bar{\alpha}) \bar{z}(\alpha-\bar{\alpha}) \tilde{z}-(\alpha+\bar{\alpha}) z(\alpha-\bar{\alpha}) \bar{z}=0$ $\forall(z, \bar{z})$. On seja, $\dot{y}$ não ó de Milnor.

Observamos que sendo $F: \mathbb{C} \rightarrow \mathbb{C}$, holomorfa $\operatorname{com} F(0)=0, \psi(z, \bar{z})=\langle F(z), z\rangle$, então det $(D \psi(z, \bar{z}))-2\left(|F|^{2}-|D F|^{2}|z|^{2}\right)$.

Vamos agora considerar o caso geral:

$$
\begin{aligned}
& F(z)=\alpha z+\gamma(z) i^{n+1} \text {, onde, } \gamma(z)=a+b z+\ldots a, a, b \in \mathbb{C}, n \geq 1 \text {. Temos, } \\
& \operatorname{DF}(z, \bar{z})=\alpha+\gamma_{z}(z) z^{n+1}+(n+1) \gamma(z) z^{n} \cdot \log 0, \\
& \operatorname{det}(D F(\tilde{z}, \bar{z}))=2\left(|F|^{2}-|D F|^{2}|z|^{2}\right) \\
& =|z|^{2}\left[-n \alpha \overline{\gamma_{z}(z)} \bar{z}^{n+1}-\alpha n \overline{\gamma(z)} \bar{z}^{n}-\bar{\alpha} \gamma_{z}(z) z^{n+1}\right. \\
& \left.-(n+1) \gamma(z) \overline{\gamma_{z}(z)}|z|^{2 n} \bar{z}-\left(n^{2}+2 n\right)|\gamma(z)|^{2}|z|^{2 n}\right] .
\end{aligned}
$$

Facilmente se verifica que se $\alpha=0$, já temos singularidade isolada na origem.

Sendo $\alpha \neq 0$, neste caso podemos tomar $\alpha=$, por um cálculo simples podemos mostrar que det $(D F(z, \bar{z})$ é uma curva analítica real passaudo pela origem. Para verificar isso basta analisar (jue det $(D F(z, \bar{z})$ tem uma reta tangente? não degenerada na origen.

Assim, podemos concluir que: $t_{F}$ é de Milnor se, e somente se, $j^{1} F(0)=0$ e $j^{n+1} F(0) \neq 0$, para algum $n \geq 2$.

Dados os campos $F_{i}, X_{i}:(\mathbb{C}, 0) \rightarrow(\mathbb{C} .0)$. Defina os campos $F:\left(\mathbb{C}^{m}, 0\right) \rightarrow\left(\mathbb{C}^{m}, 0\right)$ por $F\left(z_{1}, \ldots, z_{m}\right)=F_{1}\left(z_{1}\right) \oplus \ldots \oplus F_{m}\left(z_{m}\right)=\left(F_{1}\left(z_{1}\right), \ldots, F_{m}\left(z_{m}\right)\right) \circ X:\left(\mathbb{C}^{m}, 0\right) \rightarrow\left(\mathbb{C}^{m}, 0\right)$ por $X\left(z_{1}, \ldots, z_{m}\right)=X_{1}\left(z_{1}\right) \oplus \ldots \bigoplus X_{m}\left(z_{m}\right)$, com sua respectiva aplicaça dada por $t_{F, X}$. Temos o seguinte resultado:

Proposição 2.2.1 $\eta_{F_{1}, \mathrm{Y}}$ satisfaz a condiçäo de Minor se, e somente se, $\psi_{F_{i}, X_{i}}$ for de Milnor para cada $i=1, \ldots, m$.

Fazendo $F_{i}\left(z_{i}\right)=k_{i}, z_{i}^{a_{i}}, X_{i}\left(z_{i}\right)=z_{i}^{b_{2}}$ na Proposiçâa acima reobtemos a forma normal do Teorema 2.2 para $\sigma=I d$. 


\section{Fibração de Milnor Forte}

Como vinos anteriomente, o terema de Milnor pala singularidades reais garante que se (): $\mathbb{R}^{m}, 0 \rightarrow \mathbb{R}^{k}, 0$ lem singularidade isolada, tadmite uma fibração do tipo $\frac{\psi}{\left\|\psi^{\psi}\right\|^{\dagger}}: S^{m-1} \backslash \mathrm{N}_{K} \rightarrow S^{k-1}$, onde $\mathrm{N}_{K}$ ć uma vizinhança tubular do link $K_{\epsilon}$ na esfera $S^{m-1}$. Embora exista uma extensão $\varphi: S_{\epsilon}^{m-1} \backslash K_{\epsilon} \rightarrow S^{k-1}$ para a fibração associada à $\dot{\psi}: \mathbb{R}^{m}: 0 \rightarrow \mathbb{R}^{k}, 0$, nem sempre esta extensão é dada pela aplicação $\varphi=\frac{\psi^{k}}{\left|\dot{u}^{i}\right|}$ :

Exemplo 3.0.4 ([Mi], pag 99) :

$$
\left\{\begin{array}{l}
P=x \\
Q=x^{2}+y\left(x^{2}+y^{2}\right)
\end{array}\right.
$$

Neste exemplo, $K_{f}$ ć vazio e $\frac{\varphi}{\|t\|_{\|}}$não é uma submersäo em $S_{6}$, para todo $r>0$ suficientemente pequeno.

Neste capítulo estaremos estudando singularidades isoladas reais para as quais a aplicaçäo $\frac{v}{\left\|w_{\|}\right\|}$é a projeça do um fibrado cujo espaço total á torlo o complementar do link $K_{f}$ enl $S_{t}^{m-1}$.

Começaremos com uma definição.

Definiçāo 3.0.1 Dizemos que uma singularidade isolado real $\varphi: \mathbb{R}^{m}, 0 \rightarrow \mathbb{R}^{k}, 0$ satisfaz

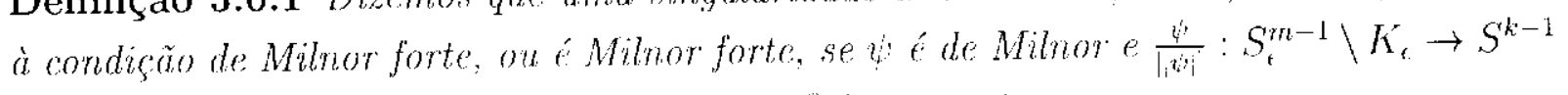
é um fibrado localmente trivial, para $\epsilon>0$ suficientemenle pequeno.

Se $:\left(\mathbb{C}^{n+1}, 0\right) \rightarrow(\mathbb{C}, 0)$ i uma funçăo holomorfa com singularidade isolada, $\psi(z)=$ $(P(z), Q(z))$, onde $P(z)$ e $Q(z)$, são respectivamente, a parte real e imaginária de $\psi$, 
scgue do Teorema da Fibração de Milnor para funçôs holomorfas que tr á Milnor forte. Exemplos nào triviais de fibraçòs que sào Nilnor forte e que näo provêm de uma estrutura. holomorfa, como visto acima, foran também obtidos em [RSV]. Vamos estudar esses cxemplos no próximo capítulo.

\subsection{Condições de Jacquemard}

Lma abordagem para estudar singularidades isoladas reais do tipo Milnor forte é descrita por A. Jacquemard [Ja] em sua tese de doutorado (veja também [Ja1]).

Seja $\psi=\left(I^{\prime}: Q\right): \mathbb{R}^{m}, 0 \rightarrow \mathbb{R}^{2}, 0$ uma aplicação analítica real com singularidade isolada na origem. Considere $\nabla P(x)$ e $\nabla Q(x)$ us gradientes de $P$ e $Q$, respectivamente. Jacquemard mostra que através do mina condição geonétrica e de uma condição algébrica, sobre as funções coordenadas da aplicaçăo to obtemos condições suficientes para que

$\frac{\psi}{\left|\psi^{*}\right|}: S_{t}^{m-1} \backslash H_{f} \rightarrow S^{1}$ seja a projeção de um fibrado localmente trivial, para $\epsilon>0$ suficientemente pequeno. Seu resultado

Teorema 3.1.1 ([Ja], Teorema 2) Seja analítica real com singularidade isolada em 0 . Suponha que exista uma vizinhança $V$ de 0 em $\mathbb{R}^{m}$ tal que:

(4) $\frac{|\langle\nabla P(x), \nabla Q(x)\rangle|}{\|\nabla P(x)\| \cdot\|\nabla Q(x)\|} \leq 1-\rho, \forall x \in V^{r}-\{0\}, 0<\rho \leq 1$.

(B) O fecho integral dos ideais gerados por $\nabla P(x)$ e $\nabla Q(x)$ no anel das funçoes analíticas reais cotncidem.

Entäo existe $c_{0}>0$, suficientemente pequeno, tal que para todo $0<\epsilon \leq c_{0}$,

$\frac{w}{\|\dot{\psi}\|}: S_{\epsilon}^{m-1} \backslash K_{\epsilon} \rightarrow S^{1}$ é a projeçäo de um fibrado localmente trivial. Além disso, esse fibrado é equivalente ao fibrado dudo por Milnor em $[\mathrm{Mi}]$.

Em [RSV] os antores modificam a condição $(B)$ de Jacquemard, trocando a condição de fecho integral pela condiçăo de fecho integral real. como na Definição 1.1.2, pag. 20, dada por Gaffincy om $[\mathrm{Ga}]$.

É fácil verificar na prova de Jaccunemard que seu resultado ainda vale so substituimos a condição $(B)$ pela condição:

$\left.\left(B_{\mathbb{R}}\right): \overline{\langle\nabla P(x)}\right\rangle_{\mathbb{K}}=\overline{\langle\nabla Q(x)\rangle_{\mathrm{R}}}$ no ancel das funçoes analíticas reais $\mathcal{A}_{m}$. 
Proposição 3.1.2 ([RSV], Proposição 3.4) Suponha que as funções componentes satisfaçam às condiçöes $(A)$ e $\left(B_{\mathrm{K}}\right)$. Entrio é é Milnor forte.

Na sequência deste traballo estaremos sempre utilizando o conceito de fecho integral real do ideal $I$. Entretanto, para simplificar a notação, indicaremos $\overline{I_{R}}$ simplesmente por $\bar{I}$.

Exemplo 3.1.3 Seja : : $\left(\mathbb{C}^{n}, 0\right) \rightarrow(\mathbb{C}, 0)$ uma aplicaçăo holomorfa com singularidade isolada. Considere $y(z)=(P(z), Q(z))$. onde $P^{\prime}(z)$ é a parte real e $Q(z)$ é a parte imaginária de $\psi$. Neste caso, usando as equaçòes de Cauchy-Riemann, verificamos diretamente que $\nabla P(x)$ e $\nabla Q(x)$ sutisfaxem as condigoes $(A)$ e $\left(B_{\text {s. }}\right)$ dadas acima. como naturalmeste era de se esperar.

No estudo da fibração de Milnor forte, um problema natural é identificar outras classes de exemplos que satisfaçam a esta condição. Neste trabalho, vamos estudar, om particular, em que condição singulariclades isoladas quasc-homogéneas satisfazem à condição Milnor forte.

A proposição abaixo é um primeiro resultado nesta direção.

Proposição 3.1.4 Seja t $-\left(I^{\prime}, Q\right):\left(\mathbb{R}^{m}, 0\right) \rightarrow\left(\mathbb{R}^{2}, 0\right)$ wma aplicaçào analítica real com singularidade isolada, tal que $\nabla P(x)$ e $\nabla Q(x)$ säo quase homogênecos do mesmo tipo e grau. Entào, existe uma vizinhança $0 \in U \subset \mathbb{R}^{m}$ tal que to satisfaz às hipóteses $(A)$ e $\left(B_{\mathbb{R}}\right)$ da Proposição 3.1 .2 , pontanto te é Milnor forte.

Demonstração. A prova da proposição segue dos Lemas 3.1.4 e 3.1 .5 abaixo.

Definição 3.1.1 Chamanos razo de Minor da aplicaçio uo maior número real $\epsilon_{0}>0$ tal que, para todo $t, 0<\epsilon \leq \epsilon_{0}, V:=1(0)$ e lranstersal a $S_{c}^{m-1}$.

Milnor prova em [Mi], pg, 17, que sendo 0 um ponto singular isolado de uma aplicação ' $\psi$, o raio de Milnor sempre cxiste.

Lema 3.1.5 Seja $t:\left(\mathbb{R}^{m}, 0\right) \rightarrow\left(\mathbb{R}^{2}, 0\right), \psi=(P, Q) . \Sigma(\psi)=\{0\}$ e suponha que $\nabla P(x), \nabla Q(x)$ sejam quase homogeneos do tipo $\left(w_{1}, u_{2}^{\prime}, \ldots, w_{m} ; d_{1}, d_{2}\right)$. Existe uma vizinhança

da origem $U \subset \mathbb{R}^{m}$ lal que: $\frac{\mid\{\nabla P(x), \Gamma Q(x)\rangle}{\| \nabla P(s) ! \cdot \nabla(Q) !(x) ! i} \leq 1-\rho, 0<\rho \leq 1$. 
Demonstração. Tome $c_{0}>0$ tal que $:^{-1}(0)$ é transversal a $S_{\varepsilon}^{m-1}$, para todo $0<\epsilon \leq \epsilon_{0}$.

Seja $U$ vizinhança de 0 no $\mathbb{R}^{m}$ tal que $\overline{D_{\epsilon_{0}}^{m}} \subseteq U$, c considere $\psi: U \rightarrow \mathbb{R}^{m}$. Como $\nabla P(x)$ e $\nabla Q(x)$ são quase homogèneos do tipo $\left(w_{1}, w_{2}, \ldots, w_{m} ; d_{1}, d_{2}\right)$, dado $x \in D_{\epsilon_{0}}^{m}-\{0\}$ existe $\lambda \in \mathbb{R}^{*}$ e $y \in S_{c_{0}-1}^{m-1}$ tais que $x=\left(\lambda^{w_{1}} y_{1}, \ldots, \lambda^{w_{n}} y_{n}\right)$.

Com efeito, sendo $x \subset D_{* 0}^{m}-\{0\}$, defind

$$
\begin{aligned}
r: & (0,1] \longrightarrow \mathbb{R}^{m}-\{0\} \\
& t \mapsto r(t)=\left(\frac{x_{1}}{t^{w_{1}}}, \ldots, \frac{x_{n}}{t^{w_{n}}}\right)
\end{aligned}
$$

Temos que $r(t)$ ó suave $o, \lim _{t \rightarrow 0}|r(t)|=+\infty$ entào, existe $\lambda \in \mathbb{R}^{*}$ tal que $r(\lambda) \cap S_{\xi_{0}}^{m-1}$ $=\mathrm{y}$.

l)efina agora,

$$
\begin{aligned}
& g: \quad S_{\varepsilon_{0}}^{m-1} \longrightarrow \mathbb{R} \\
& \quad x+g(x)=\frac{|\langle\nabla P(x), \nabla Q(x)\rangle|}{\|\nabla P(x)\| \cdot|| \nabla Q(x) \|}
\end{aligned}
$$

Como a singularidade é isolada, nos pontos onde $g$ está definida, $g$ é $C^{\infty}$.

Como $S_{\epsilon_{0}}^{m-1}$ é compacta, $g$ atinge seu valor máximo $\mathrm{cm} S_{c_{0}}^{m-1}$. Mais uma vez, como a singularidade de $\varphi$ é isolada, $\nabla P(x)$ e $\nabla Q(x)$ são linearmente indepondentes, $\forall x \neq 0$.

Assim, o máximo de $g(x)$ em $S_{\epsilon_{0}}^{m-1}$ é uma constante $M<1, \log 0 \exists 0<\rho \leq 1$ tal que $x \in S_{\epsilon_{0}}^{m-1}$ e temos $g(x) \leq 1-\rho$.

Agora, para concluir a prova do lema temos:

Para carla $x \in D_{\iota_{11}}^{m}-\{0\} \Rightarrow \exists \lambda \in \mathbb{R}^{*} \because y \in S_{t_{0}}^{m-1}$ tais que $x=\lambda y=$ $\left(\lambda^{n i_{1}} y_{1}, \ldots, \lambda^{u_{n}} y_{n}\right)$. Logo

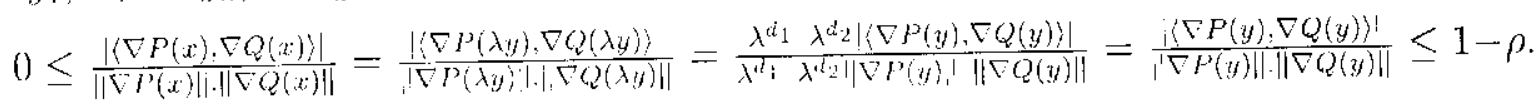

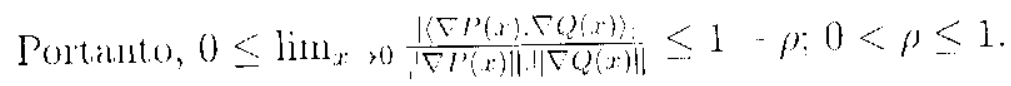

Lema 3.1.6 Suponha que $P$ e têm singularidade isolada, e que $\nabla P(x)$ e $\nabla Q(x)$ săo quase homogêneos com mesmo peso e mesmo grau. Entäo, $\overline{\langle\nabla P(x)\rangle}=\overline{\langle\nabla Q(x)\rangle}$

\section{Demonstraçāo.}

Como $\nabla P(x), \nabla Q(x)$ säo quase-homogemeos, sous poliedros de Newtom $\Gamma_{+}(\nabla P(x))$ e $\Gamma_{+}(\nabla Q(x))$ têm uma única face compacta. 
Além disso, chamando $\Delta_{1}$ e $\Delta_{2}$ as faces compactas de $\Gamma_{-}(\nabla P(x))$ e $\Gamma_{+}(\nabla Q(x))$, respectivamente, temos que das têm vertices em todos os cixos coordenados. Con efcito, seja $O x_{i}:=\left(0, \ldots, 0, x_{i}, \ldots, 0\right)$ unn cixo coordenado, e suponhamos que $x_{i}^{k} \notin \Delta_{1}, \forall k \in$ $\mathbb{N} \backslash\{0\}, \operatorname{logo} \nabla P\left(O x_{i}\right)=\nabla P\left(0, \ldots, 0, x_{i}, \ldots, 0\right)=0,0$ que contradiz a hipótese que $\nabla P(x)$ tem singularidade isolada. Portanto, $\Delta_{1}$ corta todos os eixos coordenados.

O mesmo argumento pode ser usado para $\Delta_{2}$.

Assim, $\Delta_{1}$ e $\Delta_{2}$ cortam todos os eixos coordenados.

Portanto, $\nabla P(x)$ e $\nabla Q(x)$ são Newton não degenerados conforme Definição 1.2 .5 dada no Capítulo 1 (e observaçäo do tinal deste capítulo) pois só têm uma face. Logo,

$\overline{\langle\nabla P(x)\rangle}=\left\langle\left\{x^{n} ; n \in \Delta_{1}\right.\right.$, onde $\Delta_{1}$ ć a única lace compacta de $\left.\left.\mathrm{I}_{+}(\nabla P(x))\right\}\right\rangle$ $\overline{\langle\nabla Q(x)\rangle}=\left\langle\left\{x^{k} ; k \in \Delta_{2}\right.\right.$, onde $\Delta_{2}$ é a única face compacta de $\left.\left.\Gamma_{+}(\nabla Q(x))\right\}\right\rangle$

Como estamos supondo que $\nabla P(x)$ e $\nabla Q(x)$ sân do mesmo grau con respeito aos pesos $\left(w_{1}, \ldots, w_{n}\right)$, segue que $\Delta_{1}=\Delta_{2}, \mathrm{c} \overline{\langle\nabla P(x)\rangle}=\overline{\langle\nabla Q(x)\rangle}$.

Lima consequencia imediata é:

Corolário 3.1.7 Se $\psi=(P, Q)$ é homogênca com singularidade isolada, entào t̀ é Milnor forte.

Exemplo 3.1.8 Conno um axcmplo e aplicaşà da Proposiçäo 3.1.4. seja $\psi(z, \bar{z})=\sum k_{i}, \dot{z}_{i}^{a_{i}} \cdot \bar{z}_{i}^{b_{i}}, a_{i}>1, b_{i} \geq 1 . P(z, \bar{z})$ e $Q(z, \bar{z})$, a parte real e imaginária, respectivamente de $\psi(z, \bar{z})$ e, $\nabla P(z, \bar{z}), \nabla Q(z, \bar{z})$ seus respectivos gradientes.

Temos que to é quase-homogêneo do tipo $\left(w_{1}, \ldots, u_{n}: 1\right)$, tomando $w_{j}=$ peso $x_{j}=$ peso $y_{j}, z_{j}=x_{j}+i y_{j}, w_{j}=\frac{1}{a_{j}+b_{j}}$. Portanto, segue da Proposiça 3.1 .4 que $\dot{\psi}$ o. Milnor forte.

É possivel redefinir os pesos das variaveis de modo que $\nabla P(z, \overrightarrow{\tilde{z}})$ e $\nabla(\ell(z, \bar{z})$ firquem

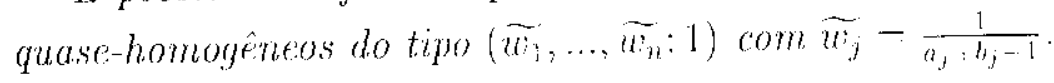

Observemos que não é possivel usar as condiçoes de Jacepuemard para cstender a Proposição 3.1.4 para incluir o caso cm que a aplicaçào $t=(P, Q)$ é quaso homogênea do tipo $\left(w_{1}, \ldots, w_{n} ; d_{1}, d_{2}\right), d_{1} \neq d_{2}$. Para isso citamos o seguinte exemplo:

Exemplo 3.1.9 Considere

$$
\left\{\begin{array}{l}
P=x \\
Q=y\left(x^{2}+y^{2}\right)
\end{array}\right.
$$


Temos que $\nabla P(x, y)=(1,0), \nabla\left(Q(x, y)-\left(2 x y, x^{2}+3 y^{2}\right)\right.$. Obviamente a condição $\left(B_{\mathbb{R}}\right)$ não está satisfeita. Por outro lado, é fácil verificar que o link é vazio e que $\varphi=\frac{\psi}{\|q\|}: S_{\epsilon} \rightarrow S^{\prime}$ é uma submersào e portanto té Milnor forte.

Com os resultarlos do próximo capítulo, será possivel estender a Proposição 3.1.4 para os germes quase homogêneos quando $d_{1}=d_{2}$. 


\section{Principais Resultados}

Vamos começar esta seçào relembrando mua construçäo devida a Seade em [S1] (ver também [S2] e [RSV]). que será muito importante para nossos objetivos.

Sejam $F, X:\left(\mathbb{C}^{n}, 0\right) \rightarrow\left(\mathbb{C}^{n}, 0\right)$ campos holomorfos com singularidade isolada da forma $F(z)=\left(k_{1} z_{\sigma_{1}}^{a_{1}}, \ldots, k_{n} \sim_{\sigma_{\sigma_{n}}}^{a_{n}}\right), X(z)=\left(t_{1} z_{1}^{b_{1}}, \ldots, t_{n} z_{n_{n}}^{b_{n}}\right), k_{i}, t_{i} \in \mathbb{C}^{*}$, como vimos no capítulo 2. Defina $\psi:\left(\mathbb{C}^{\prime \prime}, 0\right) \rightarrow(\mathbb{C}, 0)$ por $\psi(z)-\langle F(z), X(z)\rangle$, onde $\langle$,$\rangle denota o produto interno$ hermitiano. Polo Teorema 2.1.2 do Capitulo 2 , sabemos que sob certas condiçoes $\psi \psi^{\prime}$ tem singularidade isolada na origem. Seja $\pi_{\theta}: \mathbb{C} \rightarrow L_{-0}$ a projeção ortogonal de $\mathbb{C}$ sobre $L_{-\theta}$ : onde $L_{\theta}$ é a reta no plano complexo $\mathbb{C}$ passando pela origen com inclinação $-\theta$.

Defina, $\psi_{\theta}:\left(\mathbb{C}^{n}, 0\right) \rightarrow(\mathbb{R}, 0)$ por $\dot{\varphi}_{\theta}(z)=\pi_{\theta} \circ \dot{\psi}(z)$

Podemos facilmente verificar que $\iota_{\theta}(z)=\operatorname{Re}\left(\epsilon^{2 z} \omega(z)\right)$.

Como $\pi_{\theta}$ é uma submersão, cntào $t_{\theta}(z)=\pi_{\theta} \circ \ell(z)$ ć uma submersào em $U \backslash\{0\}$, para alguma vizinhança $U$ da origem. Defina $M_{\theta}: \cdots t_{\theta}^{-1}(0)$. F claro que $M_{\theta}=M_{\theta+r \pi}, \forall r \in Z$.

Lema 4.0.10 ([S2], Teorema 1.4, pg.203) (i) $\mathbb{C}^{n}=\cup_{\theta} M_{\theta}, 0 \leq \theta<\pi$.

(ii) $M=\cap_{0} M_{0}=M_{\theta_{1}} \cap M_{\theta_{2}}$, onde $M=\theta^{-1}(0), \theta_{1} \neq \theta_{2}, \theta_{1}, \theta_{2} \in[0, \pi)$.

(iii) Para cada $\theta \in[0, \pi)$ temos $M_{0}=E_{\theta} \cup M \cup F_{0+\pi}$, onde $E_{0}=\widetilde{\phi}^{-1}\left(e^{i n}\right)$ com

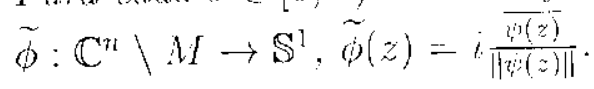

(iv) Se $\psi$ salisfaz à condica de Mhnor em 0 , cada $M_{0}^{*}:=M_{0} \backslash\{0\}$ é uma subvariedade real suave de $\mathbb{C}^{n}$ de codimensào real 1.

Vejamos o seguinte resultado. 
Teorema 4.0.11 ([RSV]) Sejam F, X campos como acima. Assuma que as sequintes condições são satisfeitas.

(a) Para cada ciclo de $\sigma$ de comprimento 1 , os correspondentes expoentes satisfazem $a_{i} \neq b_{i}$

(b) Para cada ciclo de comprimentor $>1$, rimpar, para cada $i=1, \ldots, n$, temos $a_{i} \geq 1$, $b_{i}=1$ e ao menos um dos $a_{i}$ é estritamente maior que 1.

(c) Para cada ciclo de $\sigma$ de comprimento $r>1$, r par, para cada $i=1, \ldots$, n temos $a_{i} \geq 1, b_{i}=1$ e ao menos dois $a_{i^{\prime}}$ : digamos $a_{i_{1}}$ e $a_{i_{2}}$ säo estritamente maiores que 1, com $i_{1}$ sendo impar e $i_{2}$ par no ciclo.

Entäo, í satisfaz a condiço de Minor forte em 0 . Isto é, para toda esfera $S_{\epsilon}^{2 n-1} \subset$ $\mathbb{C}^{n}$, a funçäo:

$\dot{\phi}=\frac{\dot{\varphi}}{\left\|\psi^{4}\right\|}: S_{1}^{2 n-1} \backslash \Lambda_{\mathrm{r}} \rightarrow S^{1}$ é a projeçäo de um fibrado suave, localmente trivial. Cada par de fibras antipodas o ${ }^{1}(\exp (i \theta)),-\phi^{-1}(\exp (i \theta))$, sao coladas juntas ao longo do link: $K_{c}$, formando uma (2n-2)-subvariedade suave em $S_{c}^{2 n-1}$ :

$M_{\theta} \cap S_{\epsilon}^{2 n-1}$; onde $M_{\theta}=\left\{z \in \mathbb{C}^{n} / \operatorname{Re}\langle\exp (i 0) F(z), z\rangle\right\}$. A monodromia deste fibrado é a aplicação de primeno retorno da açäo de $S^{1}$ nas variedades $M_{0_{s}}$.

A demonstração do teorema acima depende de forma essencial do fato de ty ser uma aplicação quase homogènea. Este fato ć fundanontalmente usado m toda extensão da prova. Na primeira parte da prova, para garantir que as variedales $M_{\theta_{s}}$ são transversais a todas as esferas, o depois para construir uma ação nas esferas que é transversal às fibras, conseguindo assim a trivialidade local do fibrado.

Neste capítulo. estendemos a construção de J. Seade para cqualquer aplicação 乡: $\mathbb{R}^{m}, 0 \rightarrow \mathbb{R}^{2}, 0$ com singularidade isolada, "atravós da correspondente família $\psi_{\theta}$, obtemos condição suficiente parr que to seja kilnor forte.

O resultado principal, Teorema 4.2 .2 , secào 4.2 estabelece que se a família yó (c)-regular no sentido de K. Bekka [BK], cntão ty é Milnor forte.

A conexào entre a abordagrm deste capítulo o o resultado de A. Jacquemard, [Ja] estudado no Capítulo 3, ć obtida no Teorema 4.3.1 da mesma seçäo.

Os resultados aqui apresentados fazem parte do artigo [NR], submetido para publicação. 


\subsection{Condições de Regularidade}

Como dissemos acima queremos encontrar alguma concliçâo na família to para que to seja Milnor forte. A principal referencia para esta seção é [BK]. Seja $M$ uma variedade suave e sejam $X$ o $Y$ subvariedades suaves de $M$ tais que $Y \subset \bar{X}$. 
Definição 4.1.1 ([BK]) (i) (Whitney (a)-regularidade): Dizemos que $(X, Y)$ é (a)-regular em $y_{0} \in Y$ se para cada sequência de pontos $\left\{x_{i}\right\} \rightarrow y_{0}$ tal que a sequência de espacos tangenles $\left\{W_{r_{1}}\right\}$ tende no espaco de Crassmann de dimX-planos para

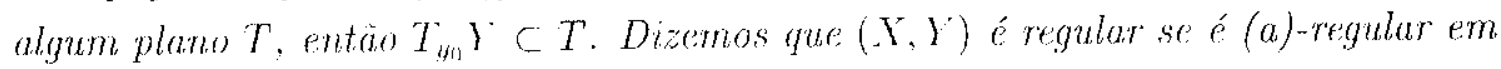
qualquer $y_{0} \in m Y$.

(ii) ((c)-regularidade):

Seja $\rho$ uma funçân nâo negativa e suave lal que $\rho^{-1}(0)=\mathrm{Y}$. Dizemos que o par $(X, Y)$ é (c)-regular em $y_{0} \in Y^{\prime}$ para uma funçäo controle $\rho$ se:

para cada sequência de pontos $\left\{x_{i}\right\} \rightarrow y_{0}$ tal que a sequência de planos

$\left\{k e r d \rho\left(x_{i}\right) \cap T_{x_{i}} X\right\}$ tende no espaço de Grassmann de (dimX-1)-planos para algum plano $T$, então $T_{y_{1}} Y^{r} \subset T$. Dizemos que o par $(X, Y)$ é (c)-regular para a função controle $\rho$ se é (c)-regular para qualquer ponto $y_{0} \in Y$ para a função controle $\rho$.

Observamos que $(c)$-regularidade $\Rightarrow(a)$ regularidade.

Vamos supor agora que $M$ lem mom métrica Riemanniana. Soja $\left(T_{Y}, \pi, \rho\right)$ uma vizinhança tubular para $Y$ junto com a projeção associada e uma função controle não negativa suave tal que $\rho^{-1}(0)=Y$ e $\nabla \rho(x) \in \operatorname{kerd} \pi(x)$.

Essa última condição indica que $\rho$ não depende do espaço de parâmetros.

Definição $4.1 .2([\mathrm{BK}])$ Dizemos que $(\mathrm{X}, \mathrm{Y})$ satisfaz à condição (m) se existe algum numero $\leftarrow>0$ tal que

$$
\begin{aligned}
(\pi, \rho) \mid X \cap T_{Y}: & X \cap T_{Y}^{c} \rightarrow Y \times \mathbb{R} \\
& x \longmapsto(\pi(x), \rho(x))
\end{aligned}
$$

é submersio, onde $T_{Y}^{t}:=\left\{x \in T_{Y} / \rho(x)<\epsilon\right\}$.

A (c)-regularidade ó então caracterizada da seguinte forma:

Teorema 4.1.1 ([BK]) O par $(\mathrm{X}, \mathrm{Y})$ é $(c)$ - regular em $y_{0} \in Y$ para a funçấo controle $)$ se e somente se o par $(X, Y)$ é $(a)$ - regular em $y_{0} \in Y$ e satisfaz condiçäo $(m)$.

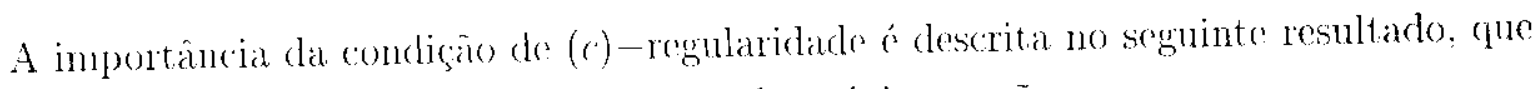
será fundanental para o principal resultado da próxima seção. 
Seja $F^{\prime}: \mathbb{R}^{m} \times \mathbb{R}, 0 \times \mathbb{R} \rightarrow \mathbb{R}, 0$ uma família a um parâmetro de germes de funçōes analíticas com singularidade isolada, $F_{t}(x)=F(x, l), X_{t}=F_{t}^{-1}(0) \backslash\{0\} \subset \mathbb{R}^{m}, 0, \forall t \in \mathbb{R}$, $X=F^{-1}(0) \backslash(0 \times \mathbb{R}) \subset\left(\mathbb{R}^{m} \times \mathbb{R}, 0 \times \mathbb{R}\right), Y=0 \times \mathbb{R}$. Então, vale o seguinte resultado:

Teorema 4.1 .2 ([Ve], $[\mathrm{KB}])$ Se o par $(X, Y)$ como acima for $(c)$ - regular, então existe uma familia de homeomorfismos $h_{t}: \mathbb{R}^{m}, 0 \rightarrow \mathbb{R}^{m}, 0$ tal que $h_{t}\left(X_{t}\right)=X_{0}$ e, $\left\|h_{t}(x)\right\|=\|x\|$ sobre $F^{-1}(0)$.

\subsection{Resultado Principal para Fibrações de Milnor Reais}

Seja $: \mathbb{R}^{m}, 0 \rightarrow \mathbb{R}^{2}, 0, \varphi(x)-(P(x), Q(x))$, uma aplicacão analítica com singularidade isolada na origem 0 . Considere $(P(x), Q(x)) \sim P(x)+i Q(x)$ a identificação natural com os números complexos. Defina $i_{\theta}: \mathbb{R}^{m}: 0 \rightarrow \mathbb{R}, 0$ dada por $\psi_{\theta}(x)=\pi_{\theta} \circ \psi(x)$. Podemos facilnnente verificar, fazendo uma mudança de base em $\mathbb{R}^{2} \equiv \mathbb{C}$. que obtemos

$$
\left.\omega_{\theta}(x)=\operatorname{Re}\left(c^{i \theta}\right)(x)\right)-\cos (\theta) P(x)-\sin (\theta) Q(x) .
$$

Defina $M=\theta^{-1}(0)$ e $M_{\theta}:=\psi_{\theta}{ }^{1}(0)$. () Lema seguinte ć uma generalização do Lema 4.0.10.

Lema 4.2.1 Seju $0 \in U \subseteq \mathbb{R}^{m}$ uma vizmhança da origem tal que para todo $x \in U \backslash\{0\}$. temos o rankin(x) seju mámo. Ention, temos:

(i) $U=\cup_{\theta}\left(M_{\theta} \cap U\right), 0 \leq \theta<\pi$.

(ii) $M=\cap_{\theta} M_{\theta}=M_{\theta_{1}} \cap M_{\theta_{2}}$, onde $M=\varphi^{-1}(0), \theta_{1} \neq \theta_{2} \theta_{1}, \theta_{2} \in[0, \pi)$.

(iii) Para cada $\theta \in[0, \pi)$ temos $H_{\theta}=E_{\theta} \cup M \cup E_{\theta \rightarrow-\pi}$. onde $E_{\alpha}=\tilde{\phi}^{-1}\left(e^{i \alpha \gamma}\right) \operatorname{com} \tilde{\phi}$ :

$U \backslash M \rightarrow \mathbb{S}^{1}$

$\widetilde{\phi}(x)=i \frac{\overline{y(x)}}{\|\psi(x)\|}$.

(iv) Se satisfaz a condiça de Minor emo. cadu $M_{0}^{*}=M_{\theta} \backslash\{0\}$ é uma subvariedade. real suave de $U \backslash\{0\}$, fommala por $E_{0} . E_{\theta ! \frac{\pi}{2}}$ de codimensäo real 1 e, $M \backslash\{0\}$ tendo codimensäo real 2 , se $M \backslash\{0\}$ for näo vazio.

Demonstração. A idéia da prova é análoga à prova do Lema 4.0.10. Vamos começar pela propriedade (iii). 
- $M \subset M_{\theta}$.

Seja $x \in M:=\left\{x \in \mathbb{R}^{m} / \hat{\varphi}(x)=0\right\}$. Então, $y_{0}(x)=\pi_{0}(\psi(x))=0$, e portanto $x \in M_{\theta}$.

- $E_{\theta} \subset M_{\theta}$.

Seja $x \in E_{\theta}=\tilde{\phi}^{-1}\left(e^{i \theta}\right)$.

Então $\tilde{\phi}(x)=\frac{\overline{i \eta(x)}}{\| \psi^{h}(x) !}=e^{i \theta}, \psi(x) \neq 0$. Portanto, $\overline{i t(x)}=\|\psi(x)\|_{i} e^{i \theta}$, on seja, $\psi(x)=$ $\|\psi(x)\| e^{i\left(-\theta+\frac{\pi}{2}\right)}$. Logo, $\psi(x) \in L_{-\theta+\frac{\pi}{2}}$, c portanto $x \in M_{\theta}$.

- $E_{\theta+\pi}^{r} \subset M_{\theta}$.

Observe que $L_{\theta}-L_{-0+\pi}\left(\pi_{0}=\pi_{0, \pi}\right.$. Assins, cste caso já está incluso no anterior. $\log O, E_{\theta} \cup M \cup E_{\theta \cdot \tau} \subseteq M_{\theta}$.

Agora, se $x \in M_{0}=\psi_{\theta}^{-1}(0)$, isto $\dot{c}, 0=\dot{\varphi}_{\theta}(x)-\pi_{\theta} \circ f(x)$ e portanto, $\psi(x) \in L_{-\theta+\frac{\pi}{2}}$, $\psi(x)=\|\psi(x)\| e^{i\left(-\theta-\frac{\pi}{2}\right)}=\|\psi(x)\| e^{-i \theta}$. Se $\psi(x)=0$, então já temos $x \in M$.

Se $\psi(x) \neq 0 \therefore e^{i \theta}=\frac{\overrightarrow{t(x)}}{\|\left.(x)(x)\right|_{1}}=\widetilde{\phi}(x) \therefore x \in \widetilde{\phi}^{-1}\left(e^{i \theta}\right)=E_{\theta} \therefore M_{\theta} \subseteq E_{\theta} \cup M \cup E_{\theta+\pi}$.

Portanto, $M_{\theta}=E_{\theta} \cup M \cup E_{\theta+\pi}$

- Para mostrar (ii), observe que do resultado anterior já temos que: $M \subseteq \cap_{\theta} M_{0}$. Para provar a inclusão contrária vanos mostrar que para qualquer $\theta_{1}, \theta_{2} \in[0, \pi), \theta_{1} \neq \theta_{2}$ temos $M_{\theta_{1}} \cap M_{\theta_{2}}=M$

Claro que $M \subseteq M_{\theta_{1}} \cap M_{\theta_{2}}$. Como $H_{\theta_{1}}=E_{\theta_{1}} \cup M \cup E_{\theta_{1}+\pi}, M_{\theta_{2}}=E_{\theta_{2}} \cup M \cup E_{\theta_{2}+\pi}$ e $E_{\theta_{1}}=\widetilde{\phi}^{-1}\left(e^{i \theta_{1}}\right), E_{\theta_{2}}=\widetilde{\phi}^{-1}\left(e^{i \theta_{2}}\right)$, temos que se $x \in E_{\theta_{1}} \cap E_{\theta_{2}} \therefore e^{i \theta_{1}}=\widetilde{\phi}(x)=e^{i \theta_{2}} \therefore$ $\theta_{1}-\theta_{2}=2 n \pi ; n \in \mathbb{Z}$. Como $\theta_{1}, \theta_{2} \in[0, \pi)$ temos $\theta_{1}=\theta_{2}$. Portanto, $E_{\theta_{1}}=E_{\theta_{2}}$. Análogo nos outros casos. Ou seja, se $\theta_{1} \neq \theta_{2}$ só podemos ter $M_{\theta_{1}} \cap M_{\theta_{2}}=M$. Podemos agora concluir que $M=\cap_{\theta} M_{0}$.

- $\cup_{0}\left(M_{\theta} \cap U\right)=U$.

Claro que $\cup_{0}\left(M_{\theta} \cap U\right) \subseteq U$.

Tome $x \in U$. Se $\psi(x)=0$, não temos nada a fazer pois $x \in M \subseteq \cup\left(M_{0} \cup U\right)$.

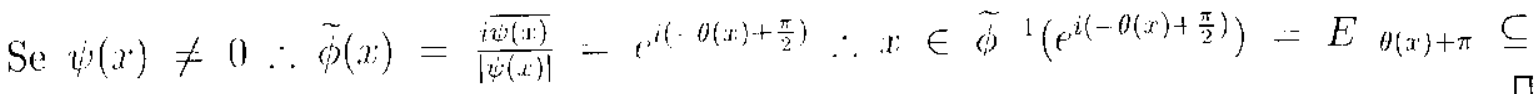
$\cup_{\theta}\left(M_{0} \cup U\right)$.

- Como é uma submersão fora da origem 0, logo é una aplicacão aberta, portanto $\psi^{-1}\left(L_{-\theta+\frac{\pi}{2}}\right)$ é llào vazio, e $\psi_{\theta}^{-1}(0)=\dot{1}^{1}\left(L_{-\theta+\frac{\pi}{2}}\right)=t^{-1}\left(L_{-\theta+\frac{\pi}{2}}^{\dagger}\right) \cup \psi^{-1}(0) \cup \psi^{1}\left(L_{-\theta+\frac{\pi}{2}}^{-}\right)$ 
é uma subvariedade suave em $U \backslash\{0\}$, pois $\psi_{0} !$ ! $\{0\}$ é também submersão, e estamos considerando $L_{-\theta+\frac{\pi}{2}}=L_{-\theta-\frac{\pi}{2}}^{+} \cup\{0\} \cup L_{-\theta+\frac{\pi}{2}}^{-}$, com $L_{-\theta+\frac{\pi}{2}}^{-}$o semi-cixo acima do eixo Oy. e $L_{\theta+\frac{\pi}{2}}$ o semi-eixo abaixo do cixo oy. Observe agora que, $y^{-1}\left(L_{-\theta+\frac{\pi}{2}}^{+}\right)=E_{\theta(x)}$, $\psi^{-1}\left(L_{-\theta+\frac{\pi}{2}}^{-}\right)^{-}-E_{\theta(x)+\pi} c b^{-1}(0)-M . E_{i \theta(x)}$ e $E_{\theta(x)+\pi}$ sâo subvariedades reais de $U$ de codimensão $1, e M \backslash\{0\}$ é subvaricdade real do $U$ de codimensão real 2 , se for năo vazio. $\square$

Podemos agora enunciar o resultado principal deste trabalho:

Teorema 4.2.2 Seja $\psi:\left(\mathbb{R}^{m}: 0\right) \rightarrow\left(\mathbb{R}^{2}, 0\right)$ um germe de aplicaçăo analítica com singularidade isolada na origem. Lal que a famtitia $\varphi_{\theta}:\left(\mathbb{R}^{m}, 0\right) \rightarrow(\mathbb{R}, 0)$ associada é $(c)$ - regular para a funçäo $\rho(x, \theta)=\sum_{i} x_{i}^{2}$. Futìo \&: Milnot Forte.

Demonstração. Supondo que $\varphi_{0}$ é (c)-regular, existe uma família $h_{\theta}:\left(\mathbb{R}^{m}, 0\right) \rightarrow\left(\mathbb{R}^{m}, 0\right)$ de germes de homeomorfismos com $h_{0}=I d r\left\|h_{\theta}(x)\right\|=\|x\|$ (preservando as esforas, ('m to $\left.{ }^{1}(0)\right)$, lal que $\psi_{y} \circ h_{0}(x)=\psi_{0}(x)$. Portanto, todos os $M_{0^{t} s}$ são homeomorfos. Como (c)-regularidade é cquivalente a (a)-regularidade e a condição (m) pelo Teorema 4.1.1, tomos em particular para a família to que existe $\epsilon>0$, suficientemente pequeno, tal que $M_{\theta} \bar{T} S_{t}^{m-1}, v \theta$. Cada $M_{\theta}$ é subvariedade de $U$, e

$M_{\theta} \cap S_{\epsilon}^{m \cdot 1}=E_{\theta} \cap S_{\epsilon}^{m-1} \cup M \cap S_{\epsilon}^{m-1} \cup E_{\theta+\frac{\pi}{2}} \cap S_{c}^{m \cdot 1}$

Pelo Lema 4.2.1 acima, temos:

(i) $S_{\mathrm{c}}^{m 1}=\cup_{0}\left(M_{\theta} \cap S_{\mathrm{c}}^{m-1}\right)$

(ii) $K_{\epsilon}:=S_{t}^{m-1} \cap M=\bigcap_{\theta}\left(M_{\theta} \cap S_{t}^{m-1}\right)$, se $M \backslash\{0\}$ for não vario.

(iii) Para cada $\theta \in[0, \pi)$ tenos

$$
M_{\theta} \cap S_{c}^{m-1}-\left(E_{\theta} \cap S_{c}^{m-1}\right) \cup\left(M \cap S_{c}^{m-1}\right) \cup\left(E_{0 ! \pi} \cap S_{c}^{m-1}\right)
$$

(iv) Cada $M_{\theta} \cap S_{\epsilon}^{m-1}$ ó uma subvariodade real da esfora $S_{\epsilon}^{m-1}$ cujo focho é $K_{\epsilon}$.

Defina a subvariedade $F_{\theta}^{\prime}:=E_{0} \cap S_{1}^{\prime \prime-1}$. Por (iii) trmos que

$$
M_{\theta} \cap S_{\epsilon}^{m-1}=F_{\theta} \cup K_{\epsilon} \cup F_{\theta+\pi}
$$

Para cada $\theta_{1}, \theta_{2} .\left(\psi_{\theta_{1}} \circ h_{\theta_{1}}\right)^{-1}(0)=\psi_{0}^{-1}(0)=\left(\psi_{\theta_{2}} \circ h_{\theta_{2}}\right)^{-1}(0)$. Ent $\tilde{a} 0, h_{\theta_{1}}^{-1}\left(\left(\psi_{\theta_{1}}\right)^{-1}(0)\right)=$ $M_{0}=h_{\theta_{2}}^{-1}\left(\left(\psi_{\theta_{2}}\right){ }^{\prime 1}(0)\right)$, e portanto, $h_{\theta_{1}}^{-1}\left(H_{\theta_{1}}\right)=h_{\theta_{2}}{ }^{-1}\left(M_{\theta_{2}}\right)$ 
Logo, $h_{\theta_{2}} \circ h_{\theta_{1}}{ }^{1}\left(M_{\theta_{1}} \cap S_{t}^{m}{ }^{\mathrm{J}}\right)-M_{\theta_{2}} \cap S_{f}^{m-1}$

É natural definir,

$$
\begin{aligned}
\Gamma_{r}: & \mathbb{R} \times S_{r}^{m}{ }^{1} \rightarrow S_{f}^{m-1} \\
& z \longmapsto h_{\theta} \circ h_{f v}^{-1}(z)
\end{aligned}
$$

para $z \in M_{i x}$.

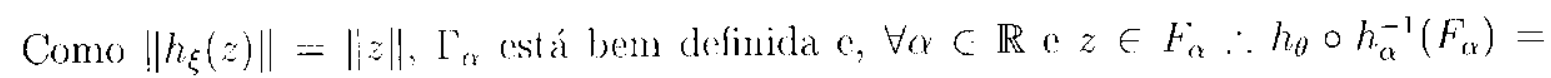
$h_{q} \circ h_{\alpha}^{-1}\left(E_{\alpha} \cap S_{i}^{m-1}\right)=h_{\theta} \circ h_{\alpha r}^{-1}\left(E_{\alpha}\right) \cap h_{\theta} \circ h_{\alpha}^{-1}\left(S_{i}^{m-1}\right)=E_{\theta} \cap S_{\uparrow}^{m-1}=F_{\theta}$

A ação I ${ }_{c}$ ć transversal as fibrass ce satisfaz

$\Gamma_{\alpha}\left(K_{t}\right)=\Gamma_{a}\left(M \cap S_{c}^{m-1}\right)=\Gamma_{a}(M) \cap S_{f}^{m-1}=M \cap S_{c}^{m-1}=K_{c}$, e portanto, deixa $o$ link invariante. Analogamente a [Mi], concluímos que $\frac{\psi}{\|\dot{\psi}\|}$ é a projeção de um fibrado localmente trivial.

Exemplo 4.2.3 A recíproca do teorema anterior nâo é verdadeira. Basta considerar a seguinte aplicaçio:

$$
\left\{\begin{array}{l}
P=x \\
Q=y\left(x^{2}+y^{2}\right)
\end{array}\right.
$$

veremos abaino que: $f:=(P, Q)$ ¿ Minor forte, mas a correspondente famíla näo é (a) - regular.

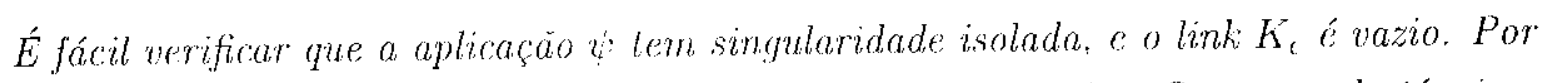
um cálculo simples lemos que $\frac{\psi}{\| \psi \psi_{i}}$ é sempre regular para loda esfera $S_{\epsilon}$. e usando técnicas de cálculo diferencial, podemos verificar que a aplicaçao $\frac{b}{\|\dot{q}\|}$ sobrejetora. Também é fácil mostrar que a famalia $\psi_{0}:=\emptyset(x, y, 0)$ associada a bem raio de Milnor infinito. On seja, satisfaz a condicäo (m). Vamos considerar abaixo uma sequência especial de pontos $p_{i}$ em. $\psi^{-1}(0)$ e mostrar que a fantilia năo é (a)-regular.

Para isso considere $p_{i}=\left(x_{i}, y_{i}, \theta_{i}\right)$, onde $x_{i} \rightarrow 0, y_{i}=0, \theta_{i}=\frac{\pi}{2}$. Claramente temos que $\psi_{0}\left(p_{l}\right)=0$, ou seja, $p_{i}$ pertence a $\mathrm{X}:=\psi_{0}{ }^{-1}(0)$. Temos que $T_{p_{i}} \mathrm{X}=\nabla \ell\left(r_{i}, y_{i}, \theta_{i}\right)^{\perp}$. Considere $y_{0}-\lim _{i} p_{i}=\left(0,0, \frac{\pi}{2}\right)$. Porem, $\lim _{i} T_{m}, X \supseteq T_{y_{0}} Y^{\prime} \therefore\left(\lim _{i} T_{p_{i}} X\right)^{\perp} \subseteq\left(T_{y_{0}} Y\right)^{\prime}$. Como $\left(T_{y_{0}} Y\right)^{\perp}=\mathbb{R}^{2} \times\{0\} e\left(\lim _{i} T_{p,} X^{\perp}=\lim _{i}\left(T_{p}, X\right)^{\perp}\right.$, temos:

$T_{p_{i}} X^{\perp}=\nabla \varphi\left(x_{i}, y_{i}, \theta_{i}\right)=\left(\cos \theta_{i}-2 x_{i} y_{i} \sin \theta_{i},-\sin \theta_{i}\left(x_{i}^{2}+3 y_{i}^{2}\right),-\sin \theta_{i} x_{i}-\cos \theta_{i} y_{i}\left(x_{i}^{2}+\right.\right.$ $\left.\left.y_{i}^{2}\right)\right)=\left(0,-x_{i}^{2}:-x_{i}\right)$. Analisando no projetivo temos, $\left(0: x_{i}^{2}: x_{i}\right)=\left(0: x_{i}: 1\right)$ que é uma 
sequência que converge para $(0: 0: 1)$. Porém, a direçăo $(0,0,1) \not \subset\left(T_{! m} Y\right)^{\perp}=\mathbb{R}^{2} \times\{0\}$. Ou seja, a Jamilia è nào é (c)-regular.

Com o Teorema 4.2.2 obtemos uma generalizaça do Teorema 4.0.11:

Corolário 4.2.4 Se $u^{\prime}:\left(\mathbb{C}^{n}, 0\right) \rightarrow(\mathbb{C}, 0)$ é quase-homogênea do tipo $\left(u_{1}, \ldots, w_{n}: d\right)$ com singularidade isolada, entäo é Milnor Forte.

Demonstração. Conı é qu guase liomogônea. já sabemos que a família to é $(c)$-regular, conforme $[\mathrm{KB}]$.

\subsection{Relação com os Resultados de Jacquemard}

Considere a famúlia

$$
\begin{aligned}
\psi_{\theta}: & \left(\mathbb{R}^{m}, 0\right) \rightarrow(\mathbb{R}, 0) \\
& x \longmapsto \operatorname{Re}\left(e^{i, \theta}(P(x)+i Q(x))\right)=\cos (0) P(x)-\sin (\theta) Q(x) .
\end{aligned}
$$

O resultado abaixo relaciona as condições de fecho integral para a família $\psi_{0}$ e as condições $(A) \oplus\left(B_{\mid *}\right)$.

Teorema 4.3.1 Srja $t=(P, Q):\left(\mathbb{R}^{m}, 0\right) \rightarrow\left(\mathbb{R}^{2}, 0\right)$. Ention, $P(x)$ e $Q(x)$ sutisfazem as

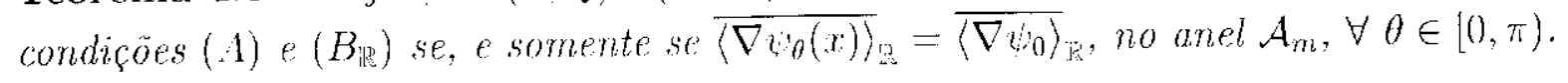

\section{Demonstraçāo.}

Seja $\gamma:\left(\mathbb{R},(0) \longrightarrow\left(\mathbb{R}^{n}, 0\right)\right.$ uma curva analítica real nào constante.

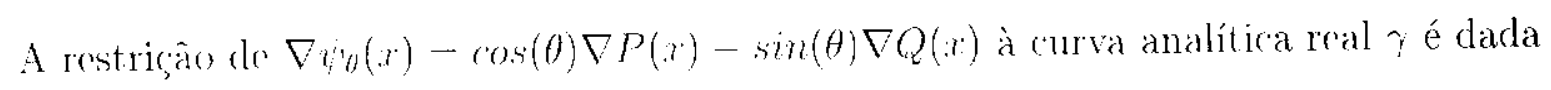
por:

$$
\nabla \psi_{\theta}(\gamma(t))=\cos (\theta) \nabla P(\gamma(t))-\sin (\theta) \nabla Q(\gamma(t))
$$

Considere os seguintes deservolvimentos de Taylor :

$$
\begin{aligned}
& \nabla P(\gamma(t))=a_{1} t^{m_{1}}+\ldots, a_{1} \in \mathbb{R}^{m}-0, n_{1} \in N . \\
& \nabla Q(\gamma(t))=b_{1} t^{m_{1}}+\ldots, b_{2} \in \mathbb{R}^{n^{n}}-0, m_{1} \in N .
\end{aligned}
$$


Como $\overline{\langle\nabla P(x)\rangle}=\overline{\langle\nabla Q(x)\rangle}$, entäa $n_{1}=m_{1}$. Logo temos

$\nabla y_{0}(\gamma(t))=\left(\cos (\theta) a_{1}-\sin (\theta) b_{1}\right) t^{n_{1}}+\ldots$

Por hipótese,

$\frac{\mid\langle\nabla P(x), \nabla Q(x)\rangle}{|\nabla P(x)||\nabla Q(x)|} \leq 1-\rho$. para algum $0<\rho \leq 1$ então, para cada $\gamma:(\mathbb{R}, 0) \longrightarrow$ $\left(\mathbb{R}^{m}, 0\right)$ analítica real temos que a parte principal satisfa\% $\frac{\mid\left\{u_{1}, b_{1} \mid\right.}{\left|a_{1}\right| b_{1} \mid}<1$, ou scja, $a_{1}$ e $b_{1}$ são linearmente independentes.

Portanto, $\cos (\theta) a_{1}-\sin (\theta) b_{1} \neq 0, \forall \theta \Longrightarrow v\left(\nabla \psi_{\theta}\right)-n_{1}=v\left(\nabla \psi_{0}\right)$, onde $v$ ća valoração canonica daula na Definição 1.1.2. Conno $\gamma$ á uma curva arbitrária. segue que $\overline{\left\langle\nabla \psi_{\theta}\right\rangle}=\overline{\langle\nabla P\rangle} \diamond$

Reciprocamente, se $\overline{\langle\nabla \hat{\psi}\rangle}=\overline{\left\langle\nabla \psi_{0}\right\rangle}=\overline{\langle\nabla P\rangle}$ então considerando $\theta=\frac{\pi}{2}$ temos a condição $\left(B_{\mathbb{R}}\right)$ r consequentemente para toda curva $\gamma:(\mathbb{R}, 0) \longrightarrow\left(\mathbb{R}^{m} ; 0\right)$ analítica real niào constante temos,

$$
\nabla \psi_{\theta}(\gamma(t))=\left(\cos (\theta) u_{1}-\sin (\theta) b_{1}\right) l^{n_{1}}+\ldots .
$$

Observe que $\forall \theta, \cos (\theta) a_{1}-\sin (\theta) b_{1} \neq 0$, caso contrário existiráa algum $\theta_{0}$ tal que $\cos \left(\theta_{0}\right) a_{1} \cdots \sin \left(\theta_{0}\right) b_{1}=0, e v\left(\nabla \eta_{0}\right)>v(\nabla P)($ absurdo! $)$

Se $\cos (\theta) \neq 0 \therefore a_{1}-\frac{\sin (\theta)}{\cos (\theta)} b_{1}=a_{1}-\tan (\theta) b_{1} \neq 0$.

De $\cos (\theta) \neq 0 \Longrightarrow \theta \neq\left(\frac{2 k+1}{2}\right) \pi, k$ inteiro, como para $\theta \in\left[0, \frac{\pi}{2}\right) \cup\left[\frac{\pi}{2}, \pi\right), \tan (\theta)$ atinge todos os valores reais e ainda temos $a_{1}-\tan (\theta) b_{1} \neq 0$ portanto $a_{1}$ e $b_{1}$ săo linearmente independentes. Como a curva $\gamma$ foi arbitrária, $\frac{\mid\langle P P(x) \cdot \nabla Q(x)|}{\nabla P(x)|\nabla Q(x)|} \leq 1-\rho$, para algum $0<\rho \leq 1$, e para $\forall x$ suficientemente próximo da origem.

Vamos mostrar agora cuo, de fato nossa condição de (c)-regularidade é mais fraca que as hipóteses de Jacquemard. Nesta direçào, vamos mostrar que as condições $(A)$ e $\left(B_{\mathbb{R}}\right)$ implicam a $(c)$-regularidade para a nossa família. Exibiremos um exemplo de singularidade isolada real, tal que a correspondente família $\psi_{\theta}$ associada ó (c)-regular, mas a singularidade nâu satisfaz as hipóteses do T'eorema de Jacquemard. Comoçaremos com uma definição de w-regularidade que pode ser encontrada em [FP].

Definição 4.3.1 Seja $F_{z}:\left(\mathbb{R}^{n}, 0\right) \rightarrow(\mathbb{R}, 0), z \in \mathbb{R}$ uma famália de aplicações. Dizemos que a familia é $w$ - regular se existe uma conslante $K>0$ tal que: $\left|\frac{\partial F(x, z)}{\partial z}\right| \leq\left. K\right|_{1} x_{1}\|\cdot\| \frac{\partial F_{\Sigma}}{\partial x} \|$, onde $\frac{\partial F_{z}}{\partial x}$ denota o gradiente da funçäo $F_{z}$. 
Lema 4.3.2 Pura a fammia $(x, t)=\cos (t) P(x)-\sin (t) Q(x)$, se $\overline{\left\langle\nabla_{x} \psi(x, t)\right\rangle_{\mathcal{A}_{n}}}=\overline{\langle\nabla P(x)\rangle_{\mathcal{A}_{n}}}$,

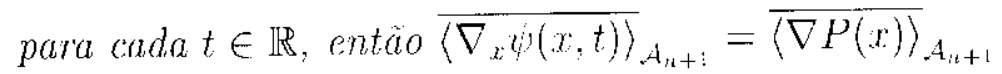

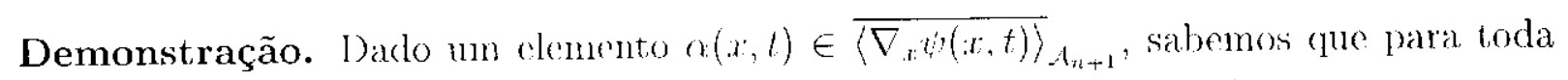
curva analítica real não constante $\gamma(s)=(x(s), t(s))$, com $\gamma(0)=(0,0)$, temos que,

$$
\begin{aligned}
\nu(\alpha \circ \gamma(s)) & \geq \nu\left(\cos (t(s)) \frac{\partial P}{\partial x_{j}}(x(s))-\sin (t(s)) \frac{\partial Q}{\partial x_{j}}(x(s))\right) \\
& \geq \min \left\{\nu\left(\cos (t(s)) \frac{\partial P}{\partial x_{j}}(x(s))\right) \cdot \nu\left(\sin (t(s)) \frac{\partial Q}{\partial x_{j}}(x(s))\right)\right. \\
& =\nu\left(\cos (t(s)) \frac{\partial P}{\partial x_{j}}(x(s)) \geq \nu\left(\frac{\partial P}{\partial x_{j}}(x(s)) \therefore{\overline{\langle\nabla} x)(x, t)\rangle_{\mathcal{A}_{n+1}} \subseteq \overline{\langle\nabla P(x)\rangle_{\mathcal{A}_{n+1}}}},\right.\right.
\end{aligned}
$$

ou podemos ter:

$$
\begin{aligned}
\nu(n \circ \gamma(s)) & \geq \min \left\{u \left(\cos (t(s)) \frac{\partial P}{\partial x_{j}}(x(s)), \nu\left(\sin (t(s)) \frac{\partial Q}{\partial x_{j}}(x(s))\right.\right.\right. \\
& =\nu\left(\cos (t(s)) \frac{\partial Q}{\partial x_{j}}(x(s)) \therefore \overline{\langle\nabla}_{x} \psi(x, t)\right\rangle_{\mathcal{A}_{n+1}} \subseteq \overline{\langle\nabla Q(x))}_{\mathcal{A}_{n+1}} .
\end{aligned}
$$

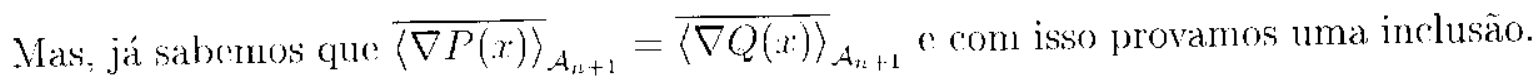

Para verificarmos que $\overline{\langle\nabla P(x)}_{\mathcal{A}_{n+1}} \subseteq \overline{\langle\nabla, x} \overline{(x, t)\rangle}_{\mathcal{A}_{t+1}}$ ó suficiente verificar que para toda curva analílica real não constants $\gamma(s)=(x(s) . t(s))$. em $\mathbb{R}^{\prime \prime} \times \mathbb{R}, 0, \gamma(0)=(0,0)$, temos.

$$
\begin{aligned}
\nu\left(\frac{\partial P}{\partial x_{i}}(x(s))\right) & \geq \min _{j}\left\{\nu e\left(\frac{\partial P}{\partial x_{j}}(x(s))\right)\right\}=\min _{j}\left\{\nu\left(\frac{\partial Q}{\partial x_{j}}(x(s))\right)\right\} \\
& =\min _{j}\left\{\nu\left(\frac{\partial P}{\partial x_{j}}+f_{1}(t) \frac{\partial P}{\partial x_{j}}+f_{2}(t) \frac{\partial Q}{\partial x_{j}}\right)(x(s), t(s))\right\} \quad \mathrm{i}, \mathrm{j}=1, \ldots, \mathrm{n}
\end{aligned}
$$

onde $f_{1}(t)=\cos (l)-1 \cdot f_{2}(t)=\sin (t)$

Lema 4.3.3 Considere a famalia $h(x, t)=\cos (t) P(x)-\sin (t) Q(x)$ como acima. Suponha

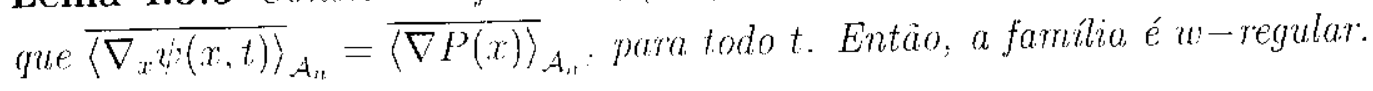

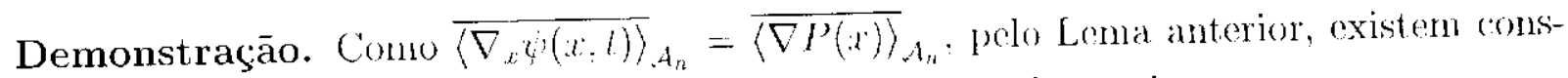
tantes $c_{1}>0, c_{2}>0, c_{3}>0 c c_{1}>0$. que não dependem de t, tais que, 
(1) $c_{1}|\|\nabla P(x)\| \leq|\left|\nabla_{x} \psi(x, t)\left\|_{1} \leq r_{2}|| \nabla P(x)\right\|: c\right.$

(2) $c_{3}\|\nabla Q(x)\| \leq\|\nabla \cdot t(x, t)\| \leq c_{1}\|\nabla Q(x)\|$.

$\log \theta$,

$$
\begin{aligned}
& \left|\frac{\partial \psi(x, t)}{\partial t}\right|=|-\sin (t) P(x)-\cos (t) Q(x)| \\
& \leq|\sin (t) P(x)|+|\cos (t) Q(x)| \leq|P(x)|+|Q(x)| \text { (desigualdade triangular) } \\
& \leq k_{1}\|x\| \cdot\|\nabla P(x)\|+k_{2}\|x\|^{\dagger}\|\nabla Q(x)\| \text { (Bochnak-Lojasicwer) } \\
& \leq k_{1}\|x\|_{1} \cdot\|\nabla P(x)\|+k_{3}\|x\| \cdot\|\nabla P(x)\| \leq K_{1}\|x\| \cdot\|\nabla P(x)\|((1) \mathrm{e}(2)) \\
& \leq K_{i}^{*} \mid x\|\cdot\| \nabla_{x} \dot{r}(x, t) \|(1) \text {. }
\end{aligned}
$$

Portanto, a familia $y(x, t)$ é $w$-regular.

Teorema 4.3.4 : Seja $\psi^{\dot{\prime}}=(P, Q):\left(\mathbb{R}^{m}, 0\right) \rightarrow\left(\mathbb{R}^{2}, 0\right)$, tal que $P(x)$ e $Q(x)$ satisfazem ass condições $(A)$ e $\left(B_{\mathbb{R}}\right)$, entío a família t: é $(c)$--regular.

Demonstração. Para o caso real e complexo, a seguinte implicaçăo sempre se verifica: $w$-regularidado $\Rightarrow(c)$-regularidado [KB]. O resultado segue portanto do lema anterior.

Exemplo 4.3.5 A recúproca do Teorema anterior nào é verdadeira. Com efeito, basta considerar a seguinte família de exemplos, para $k$ qualquer natural fixo, nüo nulo:

$$
\left\{\begin{array}{l}
P=x y \\
Q=-x^{2}+y^{2 k}
\end{array}\right.
$$

Que $f(x, y):=(P(x, y), Q(x, y))$ arina tem singularidade isolada. verifica-se facilmente. Além disso, o exemplo nào satisfaz a condiçăo $\left(B_{\mathbb{E}}\right)$.

Faremos o caso $k=2$, mas a prova no caso geral é totalmente análoga.

Considere a família $t(x, y, t)=\cos (t) x y-\sin (t)\left(-x^{2}+y^{4}\right)$, temos que o raio de Milnor é infinito. Vamos verificar a (a)-regulariclade.

Considere $\mathrm{X}=\psi^{1}(0) \backslash\{0\} \times \mathbb{R}$, e $\mathrm{I}^{\mathrm{L}}=\{0\} \times \mathbb{R}$. Seja $p_{i}=\left(x_{i}, y_{i}, 0_{i}\right)$ uma sequência qualquer en $X$ com $p_{i} \rightarrow p_{0}$, onde $p_{0} \in\{0\} \times \mathbb{R}$. Verificar a condição $(a)$ para o par $(X, Y)$ em $p_{0}$, ć o mesmo que verificar se: 


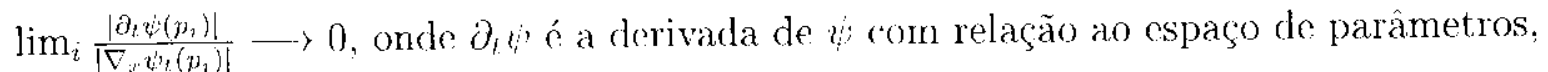
e $\nabla_{\mathrm{r}} \psi_{t}$ säo as derivadas parciais de $\varphi_{t}$ com respeito as variáveis $x_{i}, i=1, \ldots, n$.

Cono $\nabla_{x} \psi_{t}=\left(y \cos (t)+2 x \sin (t), x \cos (t)-4 y^{3} \sin (t)\right)$ e $\partial_{t} \psi^{\prime}=-\sin (t) x y-\cos (t)\left(x^{2}+\right.$ $\left.y^{4}\right)$ : porlemos usar [[RS], kema 1] para obter uma constante positiva $C_{1}$ tal que, $\left|\nabla_{x} b_{t}\right| \geq$ $C_{1}|(x, y)|$. Aplicando o mesmo resultado para $\partial_{t} t_{\text {, obtemos }}\left|\partial_{t} \psi\right| \leq C_{2}|(x, y)|^{2}$, onde $C_{2}$ é uma constante positiva. O que acaba a prova. 
Capítulo 5

\section{Famílias Analíticas Reais}

Estaremos neste capítulo interessados em famílias de gemes de aplicaçoes analíticas reais com singularidade isoladit, (uue são topologicamente triviais. Existe uma vasta bibliografia sobre o tema deste capítulo, destacaremos aqui os resultados mais relevantes para nosso estudo.

No caso complexo, condiçòes equivalentes à trivialidade topológica de famílias de germes de função com singularidade isolada, foram obtidas por vários antores, como [Te], [DG]. O seguinte teorema, devido a Greuel, reúne e estende essas caracterizaçós:

Teorema 5.0.6 ([GR], pg.161) Para qualquer deformaçào $F:\left(\mathbb{C}^{n} \times \mathbb{C}: 0\right) \rightarrow(\mathbb{C}, 0)$ de $f:\left(\mathbb{C}^{n}, 0\right) \rightarrow(\mathbb{C}, 0), n \geq 1$, uma funçuo bolomorfa, as seguintes condiçöes sào equívalentes:

(io) F é topologicamente trivial.

(i) F é uma deformaçâo $\mu$-constante de $f$, onde $\mu$ é o número de Milnor de $f$.

(ii) Para qualquer curva holomorfa

$\gamma:(\mathbb{C}, 0) \rightarrow\left(\mathbb{C}^{n} \times \mathbb{C},(0), \nu\left(\frac{\partial I}{\partial t} \circ \gamma\right)>\inf \left\{\nu\left(\frac{\partial \mu^{\prime}}{\partial x_{1}} \circ \gamma\right) / i=1, \ldots, n\right\}\right.$

(iii) A mesma afirmaçăo de (ii), trocando" $>^{\prime \prime}$ por " $\geq "$.

(iv) $\frac{\partial F}{\partial t} \in \bar{J}$, onde $J=\left(\frac{\partial F}{\partial x_{1}}, \ldots, \frac{\partial F}{\partial x_{n}}\right) \mathcal{O}_{C_{n}} \times 0$ denota o ideal jacobiano de $F$ com respeito is coordenadas $\left(x_{1}, \ldots, x_{n}\right)$. E $\bar{J}$ denota o fecho inlegral de $J$ em $\mathcal{O}_{\mathbb{C}} \times \mathbb{0}, 0$.

(v) $\frac{\partial J}{\partial t} \in \sqrt{J}$, onde $\sqrt{J}$ denota o radical de I em $\mathcal{O}_{0} \times$, 
(vi) A curva polar de $F$ com respeito a $\{t=0\}$ não se fatora, i.e.

$\left\{(x, t) \in \mathbb{C}^{n} \times \mathbb{C}, 0 / \frac{\partial F}{\partial x_{i}}=0, i=1, \ldots, n\right\}=\{0\} \times \mathbb{C}$, próximo de $(0,0)$.

Definição 5.0.2 Unu famúlia a p-parâmetros de germes $F:\left(\mathbb{R}^{m} \times \mathbb{R}^{p}, 0 \times \mathbb{R}^{p}\right) \longrightarrow$ $\left(\mathbb{R}^{k}, 0\right)$ é uma boa deformaçào, ou uma famália sem fusăo de pontos críticos, se existe uma vizinhançu $U$ de $\{0\} \times \mathbb{R}^{\prime}$ cm $\mathbb{R}^{m} \times \mathbb{R}^{\prime \prime}$ e um representante $F^{\prime}$ de $F$, tal que $F^{\prime}$ restrita a $U \cap\left(\mathbb{R}^{m}-\{0\} \times z\right)$ é uma submersaio para cadaz $z \in \mathbb{R}^{p}$. Caso contrário, dizemos que a. familia tem fusäo de pontos críticos, ou ainda que $F$ não é uma boa deformaçào.

No caso complexo o teorema de Greuel garante que para famílias de funçōes holomorfas, boa deformação é suficiente para garantir a trivialidade topológica. Uma pergunta natural é: scrá que boa deformaçào de germes no caso real implica trivialidade topológica ?

A resposta ć não. H.King $[\mathrm{HK}]$ : pg. 2, apresentia um contra-exemplo para esta pergunta, e apresenta condiçoes suficientes para qua uma boa deformaça seja topologicamente. trivial.

Definição 5.0.3 Seja $\psi_{t}:\left(\mathbb{R}^{m}: 0\right) \rightarrow\left(\mathbb{R}^{n}, 0\right), m \geq p$ uma família de aplicaçóes analúlicas com singularidade isolada, para cada $t \in \mathbb{R}$. Dizemos que a familia $\psi_{\text {, }}$ tem raio de Milnor constante, se existe $\epsilon_{0}>0$ tal que $\left(\omega_{1}^{1}(0)-\{0\}\right) 历 S_{r}^{m-1}$ para todo $t e 0<\epsilon \leq \epsilon_{0}$. Assim, $r\left(\psi_{t}\right)=\epsilon_{0}, \forall t$, e dizemos que $r\left(\psi_{t}\right)=\infty$ se $\left(\varphi_{t}^{-1}(0)-\{0\}\right) 历 S_{\epsilon}^{m} 1$ para todo $c_{0}>0$.

Em $[H K]$ temos os seguintes resultados:

Teorema 5.0.7 $([\mathrm{HK}])$ Seja $f_{z}:\left(\mathbb{R}^{m}, 0\right) \longrightarrow\left(\mathbb{R}^{k}, 0\right), z \in \mathbb{R}^{p}$ uma familia contínua de germes de aplicação polinomial, sem fusäo de pontos críticos. Seja $\delta>0$ tal que $r\left(f_{z}\right)>\delta$ para todo $z \in \mathbb{R}^{\prime \prime}$. Então, existe una familia continua de germes de homeomorfismos $h_{z}:\left(\mathbb{R}^{m}, 0\right) \rightarrow\left(\mathbb{R}^{m}, 0\right)$ tal que $f_{0}=f_{i} \circ h_{i}$.

Teorema 5.0.8 $([\mathrm{HK}])$ Seja $f_{z}^{\prime}:\left(\mathbb{R}^{\prime \prime}: 0\right) \rightarrow\left(\mathbb{R}^{k}, 0\right), z \in \mathbb{R}^{p}$, uma familia de germes sem fusao de pontos criticos e suponha que exista uma familia de germes de homeomorfismos $g_{z}:\left(\mathbb{R}^{m}, 0\right)-\rightarrow\left(\mathbb{R}^{m}, 0\right), z \in \mathbb{R}^{\prime \prime}$ tal que o germe em 0 de cada conjunto $g_{z} \circ f_{z}^{-1}(0)$ é o germe de $f_{0}^{-1}(0)$. Entio axiste uma familia de germes de homeomorfismos $h_{z}:\left(\mathbb{R}^{m}, 0\right) \longrightarrow\left(\mathbb{R}^{m}, 0\right), z \in \mathbb{R}^{p}$ e uma vizinhança $\mathrm{V}$ de $\left(0\right.$ em $\mathbb{R}^{p}$ tal que o germe em 0 de $f_{z} \circ h_{z}$ é o germe de fo paru cada z $\mathrm{E}$.

Neste capítulo estaremos preocupados com o estudo de finnílias analíticas rais cune são topologicamente triviais e mantém a condição Milnor forte ao longo das perturbaçoes da família. 


\subsection{Trivialização da Fibração de Milnor para Famílias Analíticas Reais}

No Capítulo 2 vimos que perturbaçoes sufieientemente altas de singularidades que satisfazem à condição de Milnor, também satisfazem a essa condiçăo. Fim [RSV], os autores mostram que se $h$ satisfaz as condiçoes $(A)$ e $\left(B_{\mathbb{R}}\right)$ de Jacquemard, então o mesmo acontece para perturbaçoess suficientemente altas. Um corolário deste resultado é que as condiçòes (A) e $\left(B_{\text {R }}\right)$ săo determinadas por un jato de ordem finita. Entretanto, a abordagen de [RSV] não permite obter ordens precisas para o gran de deteminação. Por exemplo, não podemos concluir que se t' é quase homogêneo e tem singularidade isolada, o gran de quase homogeneidade já determina a fibração de Milnor.

Nesta seção vamos discutir este problema. Isto pode ser feito agora de mma mancira mais eficaz, usando os resultados do Capítulo 3 e us resultados de hing [HK1].

Começaremos con uma simples observação.

Lema 5.1.1 Seja $):\left(\mathbb{R}^{m}, 0\right)-\rightarrow\left(\mathbb{R}^{2}, 0\right), \psi=(P, Q)$ uma aplicação analítica com singularidade isolada, quase homogênea do tipo $\left(u_{1}, \ldots, w_{n}, d_{1}, d_{2}\right)$. Enlão, $r(\phi)=\infty$.

Demonstração. Sabcmos que $\Sigma_{\psi}=\{0\} \Longrightarrow\left(\varphi^{-\cdots 1}(0) \backslash\{0\}\right)$ é subvariedade numa vizinhança V de 0 . Como os vetores $\nabla P(x), \nabla Q(x)$ geram o plano normal a $\left(\psi^{-1}(0) \backslash\{0\}\right)$ cm cada ponto, dizer que $\left(\psi^{-1}(0) \backslash\{0\}\right)$ não é transversal a $S_{\xi_{0}}^{m-1}$, para algum $\epsilon_{0}$, em algum ponto $x$ significa que eles se tangenciam em $x$. On seja, existem $\alpha_{3}, \alpha_{2}, \alpha_{3}$ reais nào todos nulos tais que:

$(\star): \alpha_{1} x=\alpha_{2} \nabla P(x)+\alpha_{3} \nabla Q(x)$. Defina $u x:=\left(w_{1} x_{1} \ldots, w_{n} x_{n}\right)$. Fazendo o produto rscalar cm $(\star)$ pelo campo wx, obtemos $\alpha_{1}\langle x, w x\rangle=\alpha_{2}\langle\nabla P(x), w x\rangle+\alpha_{3}\langle\nabla Q(x), w x\rangle$. Como $P(x)$ é quase homogêneo (resp. $Q(x))$ do tipo $\left(w_{1}, \ldots, w_{n} ; d_{1}\right)\left(\operatorname{resp} .\left(w_{1}, \ldots, w_{n} ; d_{2}\right)\right)$ logo pela equação de Euler temos:

$$
\langle\nabla P(x), u x\rangle=d_{1} \cdot P(x) \text { e }\langle\nabla Q(x) \cdot u x\rangle=d_{2} \cdot Q(x)
$$

Logo, $\alpha_{1} \sum_{i} w_{i} x_{i}^{2}=\alpha_{2} d_{1} \cdot P(x)+\alpha_{3} d_{2} \cdot Q(x)$.

Como $x \in\left(\psi^{\prime}(0) \backslash\{0\}\right) \cap S_{f}^{m-1}, P(x)=Q(x)=0, \log 0, \alpha_{1} \sum_{i} w_{2} x_{i}{ }^{2}=0$. Mas, $\alpha_{1} \neq 0$, pois $\nabla P(x)$ e $\nabla Q(x)$ são linearnente independentes fora de $x=0$. Logo, $\sum_{i} w_{i} x_{i}^{2}=0 \therefore x=0$.

Ou seja, vale $(\star)$ sse $x=0$, portanto, o raio de Milnor é infinito. 
Como uma conseqü̈ência do Teorema 5.0 .7 obtém-se outra demonstração para o caso $l=0$ da Proposiçào 2.1.2.

Corolário 5.1.2 ([HK]) Suponha que $f_{z}:\left(\mathbb{R}^{m}, 0\right) \rightarrow\left(\mathbb{R}^{2}, 0\right), z \in \mathbb{R}$ é uma família contínua de germes de aplicaçao polinomial quase homogêneca, com singularidade isolada. Entäo, existe uma familia contimua de germes de homeoneomorfismos $h_{z}:\left(\mathbb{R}^{m}, 0\right) \rightarrow$ $\left(\mathbb{R}^{m}, 0\right)$ lal que $f_{0}=f_{z} \circ h_{z}$.

Demonstração. Basta observar que para qualquer família quase-homogênea, com singularidade isolida, não temos fusão de pontos críticos pois vale cue $\wedge_{\mathcal{R}} f_{z}(x)=\left|d f_{z}(x)\right|^{2}=$ $\sum_{j} \mathrm{M}_{j}^{2} \geq|x|^{2 \alpha}$. pard constantes positivas $\mathrm{C}$ o $\alpha$, numa pequena vizinhanca da origem (Lema 1, [RS]).

Definição 5.1.1 Sejam $\pi: E \rightarrow B, e \pi_{1}: E_{1} \rightarrow B$ dois fibrados. Dizemos que $\pi$ é equivalente(resp. topologicamente equivalente) a $\pi_{1}$ se, existe um difeomorfismo(resp. homeomorfismo)

$h: E \rightarrow E_{1}$, tal que, $\pi=\pi_{1} \circ h$. Lsto é, o diagruma abaixo é comutativo:

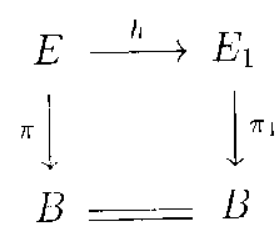

Em [HK1], King mostra que a classie do tibrado de Milnor de um germe fo, é determinada pela classe de $\mathcal{C}^{0}-\mathcal{R}$-requivalência deste germe no conjunto dos germes $f: \mathbb{R}^{m}, 0 \rightarrow \mathbb{R}^{p}, 0$ tais que, $\left.f\right|_{0 \backslash\{0\}}$ são topologicamente equivalentes a um germe de projeção, para alguma vizinhança suficientemente pecquena $\ell^{r}$ da origem. O resultado a seguir dá condiçoes suficientes para a repuivalência dos fibrados de Milnor na fimnília $F$ '.

Teorema 5.1.3 Seja $F(x, t)=(P(x, t), Q(x, t))=(f(x)+t \theta(x), g(x)+t a(x))$ uma familia de aplicaçöes analíticas. Suponha que as seguintes condiçòes se verifiquem:

(A) $\frac{\mid\left\langle\nabla P_{t}(x), \nabla Q_{t}(x)\right\rangle}{\left|\nabla P_{t}(x) \|\right| \nabla Q_{t}(x) \mid} \leq 1-\rho$, numa vizinhança $V$ de 0 em $\mathbb{R}^{n}$, paru todo $t \in \mathbb{R}$ e $0 \leq \rho<1$.

(B) ${\overline{\left\langle\nabla P_{\ell}(x)\right\rangle_{\mathcal{A}_{m}}}}-{\overline{\left\langle\nabla P_{0}(x)\right\rangle_{\mathcal{A}_{w}}}}$ ${\overline{\left\langle\nabla Q_{1}(x)\right\rangle_{\mathcal{A}_{m}}}}={\overline{\left\langle\nabla Q_{0}(x)\right\rangle_{\mathcal{A}_{m}}}}$ para todo $t \subset \mathbb{R}$. 
Enträo, a família $F_{l}$ é topologicamente trivial

\section{Demonstração.}

A idéia da prova é construir un (ampo de vetores

$V(x, t)=a(x, t) \partial_{x} P(x, t)+b(x, t) \partial_{x} Q(x, t)+\frac{\partial}{\partial t}$ (oncle $\frac{\partial}{\partial t}$ é a direção unitária do cixo $\mathrm{Ot}) \mathrm{com}$

$$
\left\{\begin{array}{l}
\langle\partial P(x, t), V(x, t)\rangle=0 \\
\langle\partial Q(x, t), V(x, t)\rangle=0
\end{array}\right.
$$

Ou seja, o campo $I$ é tangente aos níveis $X=F^{-1}(c)=P^{-1}\left(c_{1}\right) \cap Q^{-1}\left(c_{2}\right)$; onde $c=\left(c_{1}, c_{2}\right)$. Em particular para $c=0$, temos que o campo $V$ é tangente a variedade $F^{-1}(0)=P^{1}(0) \cap Q^{1}(0)$.

Dado o fluxo $\phi(x, l)$ tal que $\phi(x, 0)=x$, temos que.

$$
\frac{\partial P(\phi(x, t))}{\partial t}=\langle\partial P Y\rangle-0 \therefore P(\phi(x, t))=r t e=P(\phi(x, 0)=f(x)
$$

Análogo para $Q\left(\phi(x, t)\right.$. portanto $F^{\prime}(\phi(x, t))=F(\phi(x, 0))=(f(x), g(x))$.

Por (j.1) temos:

$$
\left\{\begin{array}{l}
\langle\partial P, 1\rangle=\left\langle\left(\partial_{x} P, \frac{\partial P}{\partial t}\right),\left(a \partial_{x} P \cdot b \partial_{x} Q, 1\right)\right\rangle=a_{i} \mid \partial_{x} P \|^{2}+b\left\langle\partial_{x} P, \partial_{x} Q\right\rangle+\frac{\partial I}{\partial t}=0 \\
\langle\partial Q, 1\rangle=\left\langle\left(\partial_{x} Q, \frac{\partial Q}{\partial t}\right),\left(a \partial_{x} P+b \partial_{x} Q, 1\right)\right\rangle=a\left\langle\partial_{x} P, \partial_{x} Q\right\rangle+\left.b\right|_{i} \partial_{x} Q \|^{2}+\frac{\partial Q}{\partial t}=0
\end{array}\right.
$$

A matriz associada ao sistema acima é:

$$
\left(\begin{array}{cc}
\left\|\partial_{x} P\right\|^{2} & \left\langle\partial_{x} P, \partial_{x} Q\right\rangle \\
\left\langle\partial_{x} P, \partial_{x} Q\right\rangle & \left\|\partial_{x} Q\right\|^{2}
\end{array}\right)\left(\begin{array}{l}
a \\
b
\end{array}\right)=-\left(\begin{array}{c}
\theta(x) \\
\alpha(x)
\end{array}\right)
$$

Seu determinante

$$
\Delta(x, t):=\left\|\partial_{x} P(x, t)_{\|}^{\prime}\right\|^{2}\left\|\partial_{x} Q(x, t)\right\|^{2}-\left\langle\partial_{x} P(x, t), \partial_{x} Q(x, t)\right\rangle^{2}
$$

satisfaz à condiçào $\Delta(x, t) \neq 0$ para todo $x \in V$, nunna vizinhança de $0 \in \mathbb{R}^{m}, \forall t$, pela hipótese (A) do trorema. Logo, aplicando a inversa nos dois lados do sistema acima temos: 


$$
\left(\begin{array}{l}
a \\
b
\end{array}\right)=-\frac{1}{\Delta}\left(\begin{array}{cc}
\left\|\partial_{x} Q\right\|_{1}^{2} & -\left\langle\partial_{x} P, \partial_{x} Q\right\rangle \\
\cdots\left\langle\partial_{x} P, \partial_{x} Q\right\rangle & \left\|\partial_{x} P\right\|^{2}
\end{array}\right)\left(\begin{array}{l}
\theta(x) \\
\alpha(x)
\end{array}\right)
$$

Portinto,

$$
\left\{\begin{array}{l}
a=-\frac{1}{\Delta}\left(\mid \partial_{x} Q \|^{2} \theta(x)-\left\langle\partial_{x} P, \partial_{x} Q\right\rangle \alpha(x)\right) \\
b=-\frac{1}{\Delta}\left(\left\|\partial_{x} P\right\|^{2} \alpha(x)-\left\langle\partial_{x} P, \partial_{x} Q\right\rangle \theta(x)\right)
\end{array}\right.
$$

Este campo está bem definido e c suare muma vizinhança aborta $U \subset \mathbb{R}^{m} \times \mathbb{R} \backslash(\{0\} \times \mathbb{R})$. Nos pontos $(0, t)$. tomos $\Delta(0, t)=0$. para garantir que o campo está bem definido no cixo $O t$, e também a unicidade do fluxo, é suficicnte garantir que existe uma constante $C^{\prime}>0$ tal que:

$$
\left\|v(x, t)-\frac{\partial}{\partial t}\right\| \leq C\|x\|
$$

Nesta direçào provaremos o seguinte resultado.

Lema 5.1.4 (al) $|\theta(x)| \leq c_{1}\|x\|_{i}\left\|\partial_{x} P\right\|$, para alguma constante $r_{1}>0$

(b) $|\alpha(x)| \leq c_{2} \mid x\|\| \partial_{x} Q \|$, para alguma constante $c_{2}>0$.

\section{Demonstração.}

$(a)$ : Como a função $\theta(x)$ é analítica e $\theta(0)=0$, temos pela desigualdade de BochnakLojasiewicz $[\mathrm{KB}]$,

$$
\left|\theta(x)_{i} \leq c_{0}\right| \dot{i}|x||| \partial_{x} \theta(x)||
$$

Por outro lado, usando a desigualdade triangular temos:

$|t|\left\|\partial_{x} \theta\right\|-\left\|\partial_{x} f(x)\right\|^{\leq} \leq \mid \partial_{x} f(x)+\partial_{x} \theta(x)\left\|_{i}=\right\| \partial_{x} \rho\left\|\leq c_{1}\right\| \partial_{x} f(x) \|_{1}^{\prime}$, onde na últina desigualdade usanos a condiçao (B), da igualdarle do fecho integral.

Agora temos,

$|t|\left\|\partial_{x} \theta\right\| \leq\left(1+c_{1}\right)\left\|\partial_{x} f(x)\right\| \leq r_{2} \|\left.\partial_{x} P(x, t)\right|_{\text {, onde novamente usamos a condição }(B)}$ na última desigualdade. 
Como vale a desigualdade para todo t. cm particular para $t=1$ temos,

$$
\left\|\partial_{r} \theta(x)\right\|^{\prime} \leq c_{2}\left\|\partial_{r} P(x, l)\right\|
$$

De $(5.7)$ ( (5.8) temos:

$\left.\|\theta(x)\| \leq c_{0}\|x\| \| \partial_{x}\right)(x)\left\|\leq\left. c^{\prime}|x \| \cdot| \partial_{x} P(x)\right|_{1}\right.$.

Da mesma forma consegue-se mostrar a parte (b).

Proposição 5.1.5 $\left\|\mathrm{V}(x, t)-\frac{\partial}{\partial t}\right\| \leq r\|\dot{x}\|$

Demonstração.

$$
\begin{aligned}
\left\|V(x, t)-\frac{\partial}{\partial t}\right\| & =\left\|a(x, t) \partial_{x} P(x, t)+b(x, t) \partial_{x} Q(x, t)\right\| \\
& \leq\left\|a(x, t) \partial_{x} P(x, t)\right\|+\left\|b(x, t) \partial_{x} Q(x, t)\right\|
\end{aligned}
$$

Vamos tentar estimal a parcela

$$
\left\|u(x, l) \partial_{x} P(x, t)\right\|
$$

Como

$\left\|a(x, t) \partial_{x} P(x, t)=|a(x, t)| \cdot|\cdot| \partial_{x} P(x, t)\right\|_{i}$.

Por (j.5) temos.

$$
\begin{aligned}
& \left|\frac{1}{\Delta}\left(\|\left.\partial_{x} Q\right|_{1} ^{2} \theta(x)-\left\langle\partial_{x} P, \partial_{x} Q\right\rangle \alpha(x)\right)\right| \cdot \| \partial_{x} P(x, t) \mid \leq \\
& \frac{1}{|\Delta|}\left\|\partial_{x} Q\right\|^{2}\left|\theta(x) i\left\|\partial_{x} P \dagger+\frac{1}{|\Delta|}\left|\left\langle\partial_{x} P, \partial_{x} Q\right\rangle\right| \cdot|\alpha(x)| i \mid \partial_{x} P\right\|\right.
\end{aligned}
$$

Mats,

$$
\begin{aligned}
& \frac{1}{|\Delta|}\left\|\partial_{x} Q\right\|_{1}^{2} \theta(x)|\cdot| \partial_{x} P \| \cdot\left(\frac{\mid \partial_{x} P\left\|^{2}\right\| \partial_{x} Q \|^{2}}{\left\|\partial_{x} P^{2}\right\|^{2}\left\|\partial_{x} Q\right\|^{2}}\right)= \\
= & \frac{|\theta(x)|}{\left\|\partial_{x} P\right\|}\left(\frac{\left\|\partial_{x} P(x, t)\right\|^{2}\left\|_{i} \partial_{x} Q(x, t)\right\|^{2} \cdots\left\langle\partial_{x} P(x, t), \partial_{x} Q(x, t)\right\rangle^{2}}{\left\|\partial_{x} P\right\|^{2}\left\|\partial_{x} Q\right\|^{2}}\right){ }^{1}= \\
= & \frac{|\theta(x)|}{\left\|\partial_{x} P\right\|}\left(1-\frac{\left\langle\partial_{x} P(x, t), \partial_{x} Q(x, t)\right\rangle^{2}}{\left\|\partial_{x} P\right\|^{2}\left\|\partial_{x} Q Q\right\|^{2}}\right)^{-1}
\end{aligned}
$$


Pela condição (A), temos que existe $\delta, 0<\delta<1$ tal que?

$\frac{\left|\left\langle\nabla P_{t}(x), \nabla Q_{t}(x)\right\rangle\right|^{2}}{\left|\nabla P_{t}(x)\right|^{2} \mid \nabla Q_{t}(x)^{2}} \leq \delta$

$$
1-\frac{\left|\left\langle\nabla P_{t}(x), \nabla Q_{t}(x)\right\rangle\right|^{2}}{\left|\nabla P_{t}(x)\right|^{2}\left|\nabla Q_{t}(x)\right|^{2}} \geq 1-\delta
$$

Do Lema 5.1.4(a), temos,

$$
\frac{\mid \theta(x) !}{\left\|\partial_{x} P\right\|} \leq c \mid 3\|x\|
$$

De (5.11) e (5.12) temos:

$$
\frac{1}{\mid \Delta_{i}}\left\|\partial_{r} Q\right\|^{2} \mid \theta(x) i\left\|_{1} \partial_{x} P\right\| \leq c_{4}\|x\|
$$

Analogamente para a parcela

$\frac{1}{\mid \Delta !}\left|\left\langle\partial_{x} P, \partial_{x} Q\right\rangle\right| \cdot{ }^{\prime} \alpha(x)|\cdot|\left|\partial_{x} P\right| \mid$ temos,

$$
\begin{aligned}
& \frac{1}{|\Delta|}\left|\left\langle\partial_{x} P, \partial_{x} Q\right\rangle\right| \cdot|\alpha(x)| \cdot\left\|\partial_{x} P\right\| \cdot\left(\frac{\left\|\partial_{x} P\right\|^{2}\left\|\partial_{x} Q\right\|^{2}}{\left\|\partial_{x} P\right\|\left\|^{2}\right\| \partial_{x} Q \|^{2}}\right) \\
& =\frac{\left|\left\langle\partial_{x} P, \partial_{x} Q\right\rangle \| \alpha(x)\right|}{\left\|\partial_{x} P\right\| ! \mid \partial_{x} Q\|\| \partial_{x} Q \|}\left(\frac{l_{x} \partial_{x} P(x, l)\left\|\left.^{2}\right|_{i} \partial_{x} Q(x, t)\right\|^{2}-\left\langle\partial_{x} P(x, t), \partial_{x} Q(x, t)\right\rangle^{2}}{\left\|\partial_{x} P\right\|^{2}\left\|\partial_{x} Q\right\|^{2}}\right)^{\prime} \\
& =\frac{\left|\left\langle\partial_{x} P, \partial_{x} Q\right\rangle \|_{\alpha}(x)\right|}{\left\|\partial_{x} P\right\|\left\|\partial_{x} Q\right\|_{\|} \partial_{x} Q \|}\left(1-\frac{\left\langle\partial_{x} P(x, t), \partial_{x} Q(x, t)\right\rangle^{2}}{\left\|\partial_{x} P\right\|^{2}\left\|\partial_{x} Q\right\|^{2}}\right)^{-1}
\end{aligned}
$$

Mas,

$$
1-\frac{\left|\left\langle\nabla P_{l}(x), \nabla Q_{t}(x)\right\rangle\right|^{2}}{\left|\nabla P_{l}(x)\right|^{2}\left|\nabla Q_{l}(x)\right|^{2}} \geq 1-\delta
$$

e, pelo Lema 5.1.4(b) e a condição $(A)$ :

$$
\frac{\left|\left\langle\partial_{x} P, \partial_{x} Q\right\rangle\right| i|\alpha(x)|}{\left\|\partial_{x} P\right\|_{\|}\left\|\partial_{x} Q\right\|\left\|\partial_{x} Q\right\|}<c \mid\|x\|_{i}
$$

Portanto de $(5.13)$ a $(5.16)$ tenus a seguinte estimativa para $(5.9)$. 


$$
\left\|a(x, t) \partial_{x} P(x, t)\right\|<c_{s}\|x\|
$$

Analogamente mostra-se que

$$
\left\|b(x, t) \partial_{x} Q(x, t)\right\|<c_{6}|| x \|
$$

Considerando $\frac{c}{2}:=\max \left\{c_{s}, c_{f}\right\}$, segue o resultado.

Corolário 5.1.6 Considere uma familia $F(x, t)$, como no teorema anterior. Se a apli-

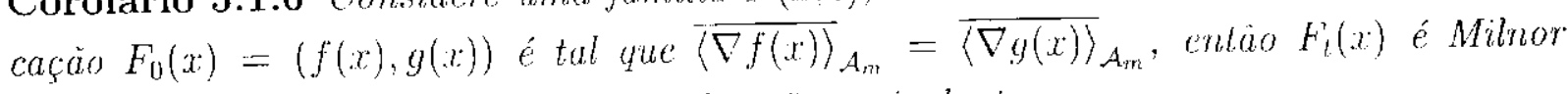
Forte, para cada t, fixo, etodos os fibrudos siio equivalentes.

Corolário 5.1.7 Considere $f_{0}=\left(P_{0}, Q_{0}\right)$ com singularidade isolada, $P_{0}$ e $Q_{0}$ satisfazendo as condiçós $(A)$ e $\left(B_{\mathrm{B}}\right)$. Suponha que $\mathrm{I}_{+}\left(\nabla P_{0}(x)\right)$ e $\mathrm{l}_{+}\left(\nabla Q_{0}(x)\right)$ sấo Newton nào degenerados. Seja $F(x, t)=\left(P_{0}(x)+t \theta(x) . Q_{0}(x)+t \alpha(x)\right)$ urma deformaçào com $\Gamma_{+}(\nabla \theta(x)) \subset \Gamma_{+}\left(\nabla P_{0}(x)\right)$ e $\Gamma_{+}(\nabla \alpha(x)) \subset \Gamma_{-}\left(\nabla Q_{0}(x)\right)$. Então, $F$ é topologicamente trivial e os fibrados sio equivalentes.

Demonstração. Que $F$ ć topologicanente trivial já segue do resultado anterior. De fato, como os poliedros de Newton $L_{+}\left(\nabla P_{0}(x)\right)$ e $\Gamma_{+}\left(\nabla Q_{0}(x)\right)$ são não-degenerados, e as deformaçoes são acima do poliedro de Newton, segue do Teorema 1.2.2 [Ma] capítulo 1 ,

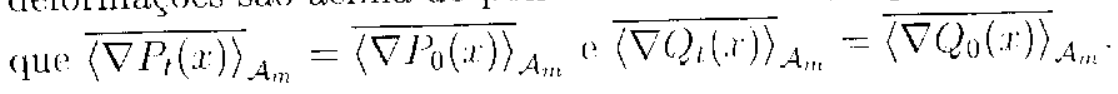

Vamos verificar que para cada t, fixo, Fi é Milnor Forte.

Para isso. considere $r: \mathbb{R}, 0 \rightarrow \mathbb{R}^{m, 0}$, una curva analítica real. conn $(x(0)=0$, não constante.

Logo. para cada t, fixo, temos:

$$
\begin{aligned}
& \nabla_{x} P_{l}(r(s))=\nabla_{x} P_{0}(r(s))+\nabla_{x} R(r(s)) \\
& \nabla_{x} Q_{t}(r(s))=\nabla_{x} Q_{0}(r(s))+\nabla_{x} S(r(s))
\end{aligned}
$$

Tomando o desenvolvimento em Taylor obtemos,

$$
\begin{aligned}
& \nabla_{x} P_{t}(r(s))=\alpha_{1} s^{a_{1}}+\ldots+\left(r_{2} s^{n_{1}}+\ldots\right. \\
& \nabla_{x} Q_{t}(r(s))=\beta_{1} s^{a_{1}}+\ldots+\beta_{2} s^{n_{2}}+\ldots
\end{aligned}
$$


Como $\Gamma_{+}\left(\nabla P_{0}(x)\right)$ e $\Gamma_{+}\left(\nabla Q_{0}(x)\right)$ são Vewton não -logencrados, cntão $a_{1} \leq n_{1} \mathrm{e}$ $a_{1} \leq n_{2}$, para toda curva analítica real.

Logo,

$$
\begin{aligned}
& \frac{\left|\left\langle\nabla P_{t}(r(s)), \nabla Q_{t}(r(s))\right\rangle\right|}{\left|\nabla P_{t}(r(s))\right|\left|\nabla Q_{t}(r(s))\right|}=\frac{\left|\left\langle\alpha_{1} s^{a_{1}}+\ldots . \beta_{1} s^{a_{1}}+\ldots\right\rangle\right|}{\left|\alpha_{1} s^{a_{1}}+\ldots\right|\left|\beta_{1} s^{a_{1}}+\ldots\right|}=\frac{\left|\left\langle\alpha_{1}, \beta_{1}\right\rangle\right| s^{2 a_{1}}+\ldots}{s^{2 a_{1}}\left|\alpha_{1}\right|(1+\ldots)\left|\beta_{1}\right|(1+\ldots)} \\
= & \frac{\left|\left\langle\alpha_{1}, \beta_{1}\right\rangle\right| s^{2 a_{1}}(1+\ldots)}{\left|\alpha_{1}\right|\left|\beta_{1}\right| s^{2 a_{1}}(1+\ldots)}=\frac{\left|\left\langle\alpha_{1}, \beta_{1}\right\rangle\right|}{\left|\alpha_{1}\right|\left|\beta_{1}\right|}+s(\ldots)=\frac{\left|\left\langle\alpha_{1}, \beta_{1}\right\rangle\right|}{\left|\alpha_{1}\right|\left|\beta_{1}\right|}+u(s)
\end{aligned}
$$

Como $\frac{\left|\left\langle\alpha_{1}, \beta_{1}\right\rangle\right|}{\left|\alpha_{1}\right|\left|\beta_{3}\right|}<1$ e $\lim _{s \sim 0} u(s)-0$, logo para s suficientemente pequeno temos:

$\frac{\mid\left\{\nabla P_{t}(r(s)), \nabla Q_{t}(r(s)) \mid\right.}{\left|\nabla P_{t}(r(s))\right|\left|\nabla Q_{t}(r(s))\right|} \leq 1-\rho(t)$ onde $0<\rho(t) \leq 1$ para $t \in \mathbb{R}$ fixo. Logo, existe uma vizinhança $V_{t} \varsubsetneqq \mathbb{R}^{m !}: 0 \in V_{t}$ tal que

$$
\forall x \in V_{t}-\{0\}: \frac{\left|\left\langle\nabla P_{t}(x)\right), \nabla Q_{t}(x)\right\rangle \mid}{\left|\nabla P_{t}(x)\right|\left|\nabla Q_{t}(x)\right|} \leq 1-\rho(t) ; 0<\rho(t)<1 .
$$

Conseqüentemente o resultado.

Corolário 5.1.8 sejam $f_{0}(x)=\left(P_{0}(x), Q_{0}(x)\right)$ e $F(x, t)=\left(P_{0}(x)+t \theta(x), Q_{0}(x)+t \beta(x)\right)$ uma deformaçào de $f_{0}$, e suponha que $\Gamma_{+}\left(\nabla P_{0}(x)\right)$ e $\Gamma_{+}\left(\nabla Q_{0}(x)\right)$ säo Newton näodegenerados.

(1) Se $f_{0}$ satisfaz à condiçăo (A) de Jaquemard, entäo F é lopologicamente trivial.

(2) Se além disso, $f_{0}$ satisfaz às condiçoes $(4)$ e $\left(B_{\mathbb{F}_{2}}\right)$, entäo $F_{t}$ é. Milnor forte para cada t e as fibraçöes associadas sùo equivalenles.

Observamos que o resultado acima pode ser estendido para deformaçóes mais gerais do tipo $F(x, t)=\left(P_{0}(x)+t \theta(x, t), Q_{0}(x)+t a(x, t)\right)$ com as mesmas hipóteses, isto é:

(A) $\frac{\left|\left\langle\nabla P_{t}(x), \nabla Q_{r}(x)\right\rangle\right|}{\left|\nabla P_{t}(x)\right| \nabla Q_{t}(x) \mid} \leq 1-\rho$, numa vizinhanga $0 \in V \subset \mathbb{R}^{m}$, para todo $l \in \mathbb{B} \rho 0 \leq \rho<1$.

(B) ${\overline{\left\langle\nabla P_{0}(x)\right\rangle_{\mathcal{A}_{m}}}}=\left\langle\overline{\nabla\left(Q_{0}(x)\right\rangle_{A_{m}}}\right.$

(C) $\mathrm{l}_{+}\left(\nabla P_{0}(x)\right)$ e $\Gamma_{+}\left(\nabla Q_{0}(x)\right)$ são Newton náa -degencrados

A trivialidade topológica da fimmília scyue do Teorema 4.9 [.JD] do Damon ou do Teorema 4.6[Ga] de Gaffney. Ambos garantem que se o diagrama de Newton do 
gradiente de $P_{0}$ e $Q_{0}$ são Newton não-degenerados, no sentido de Khovanskii [KIT], então $F$ é $\mathcal{C}^{0}-\mathcal{K}$ - topologicamente trivial.

Portanto os conjuntos dos zeros $F_{t}^{-1}(0)$ são homeomorfos. Por outro lado, é fácil mostrar que as hipóteses acina inplicam que $F$ é uma boa deformação. Iosta forma podemos usar o Trorema do King para obter a trivialidade topológica.

() resto da clemonstraçăo, isto ó, a prova de que $P_{t}$ \& $Q_{t}$ satisfazem $(A)$ \& $\left(B_{\mathrm{B}}\right)$, para todo t, segue análogo à prova que fizemos no Corolário 5.1.7 .

Proposição 5.1.9 Seja $f^{\prime}(x, u)-\left(P_{u}(x)+G(x, u), Q_{0}(x)+H(x, u)\right)$ uma deformaçà de um germe quase-homogeneo $f(x)-\left(P_{0}(x), Q_{0}(x)\right)$, do tipo $\left(w_{1}, \ldots, w_{n} ; d\right)$ com singularidade isolada, se $\int i l\left(G_{u}\right) \geq \operatorname{fil}\left(P_{0}\right)$, fill $\left(H_{u}\right) \geq \operatorname{fil}\left(Q_{0}\right)$, então $F_{u}$ a Milnor forte para todo $u$ suficientemente pequeno e as fibracoes associadas sào equivalentes. Se fil $\left(G_{u}\right)>$ fil $\left(P_{0}\right)$, fill $\left(H_{u}\right)>$ fill $\left(Q_{0}\right)$, o mesmo se verifica para todo $u \in \mathbb{R}$.

Demonstração. Como já vimos $\psi_{u}(x, \theta)=\cos (\theta)\left(P_{0}(x)+G(x, u)\right)+\sin (\theta)\left(Q_{0}(x)+\right.$ $H(x, u)) \dot{c}(c)$-regular em 0 . Portanto as aplicaçóes $F_{n}$ săo Milnor Forte para cada $u$. Além disso, a perturbaçàn ó topologicanente trivial e portanto os fibrados sào equivalentes.

\subsection{Observações finais}

Un problema importante näo considerado neste trabalho ó o estudo da topologia das singularidades definidas por um germe to com singularidade isolada que satisfaz a uma conclição Milnor forte. Aparentemente ainda não existe um método para tratar desta questäo em geral. Lm problema inportante, neste contexto, é deteminar so estas singulariclades são, ou näo. topologicamente erguivalentes a uma singularidade isolada de mua funça holomorfa. Um primeiro exemplo de uma singularidade isolada nāo equivalente a uma holomorfa foi apresentado por A' Campo $[\mathrm{C}]$.

Exemplo 5.2.1 $f: \mathbb{C}^{m+2} \rightarrow \mathbb{C}$, dada por $f\left(u, v, z_{1}, \ldots, z_{m}\right)=u v(\bar{u}+\bar{v})+z_{1}^{2}+\ldots+z_{m}^{2}$

Sejam $f: \mathbb{C}^{m+1}, 0 \rightarrow \mathbb{C}, 0$ um gerne de função holomorfa, $\phi=\frac{f}{\|\left. f\right|_{1}}: S, \rightarrow S^{1}$ a filoração de Milnor associatla, $F_{\theta}=\phi^{-1}(\exp (i \theta))$ uma fibra clesta fibraça $e h: F_{\theta} \rightarrow F_{\theta}: 0$ homeonorfismo característico da fibração o. 
O automorfisno induzido $h_{*}-\left(h^{q}\right)_{q}>0: H^{q}\left(F_{0}, \mathbb{C}\right) \rightarrow H^{q}\left(F_{0}, \mathbb{C}\right)$ ć a monodromia (a conficientes complexos) de $f^{-1}(0) \mathrm{cm}(0$.

O resultado principal de A'Campo em [C! ó o seguinte,

Teorema 5.2.2 Se o éponto singular de $f$ entäo o número de Lefschetz de h, $\Lambda(f)=\sum_{i}(-1)^{i} \operatorname{tr}\left(h_{i}\right)=0$.

Quando 0 é um pouto singular isolado de $f$, sabemos (ver [Mi]) que:

$$
H^{q}\left(F_{0}, \mathbb{C}\right)=\left\{\begin{array}{l}
\mathbb{C}: q=0 \\
0, q \neq 0, q \neq m \\
\mathbb{C}^{\mu}, q=m .
\end{array}\right.
$$

onde $\mu=\operatorname{dim} \frac{o_{m+1}}{\langle J f\rangle}$, e Jf é o ideal jacobiano de $f$. Neste caso, o teorema acima diz que $\operatorname{tr}\left(h^{m}\right)=(-1)^{m+1}$. Para o exemplo acima. A Campo mostra que $\operatorname{tr}\left(h^{m+1}\right)=2 \cdot(-1)^{m+2} \neq$ $(-1)^{m+2}$ e portanto, $\bigwedge(f) \neq 0$ of não pode ser topologicancmte equivalente a um germe holomorfo.

O cálculo da monodromia para as lamílias estudadas em [RSV é um problema ainda não abordado

Quando $\varphi: \mathbb{R}^{m}, 0 \rightarrow \mathbb{R}^{2}, 0$ é lada por $\varphi_{F, X}$, com $F, X$ campos holomorfos dados por $F(z)=\left(z_{1}^{a_{1}}, \ldots, z_{m}^{a_{m}}\right), X(z)=\left(z_{1}^{b_{1}}, \ldots, z_{m}^{b_{m}}\right), a_{i}>b_{i} \geq 1$, para tollo $i$, em [RSV] os autores provam o seguinte:

Teorema 5.2.3 ([RSV], pag 211) A variedade singular $\widehat{\mathrm{T}}=\psi^{\prime}(0)$ é homeomorfa a variedade de Bricstom $V_{1, \ldots, m}$, e as comrespondentes fibrasòes de Milnor säo topologicamente equivalentes. Mais precisamente. existe um homeomorfismo $h: \mathbb{C}^{m}, 0 \rightarrow \mathbb{C}^{m}, 0$ tal que $\psi=f \circ$ o h, onde $\int$ é o polinômio de Pham-Brieskorn $: f\left(z_{1}, \ldots, z_{m}\right)=z_{1}^{c_{1}}+\ldots+z_{m}^{c_{m}}$.

A prova é diretal. Basta combiderar $E \subset \mathbb{C}^{m}$ o divisor $\left\{z_{1} z_{2} \ldots z_{n}=0\right\}$, e $h: \mathbb{C}^{m} \rightarrow \mathbb{C}^{m}$, $h\left(z_{1}, \ldots z_{m}\right)=\left(w_{1}, \ldots u_{m}\right)$, ondo $w_{i}=\left|z_{i}\right|_{1}^{\frac{2 b_{2}}{a_{i}} z_{i}}$. Então, $h$ c claramente um difeomorfismo analítico real que se estende a um homomorfismo de $\mathbb{C}^{m}$ sobre si mesmo. Além disso, para cada $i=1, \ldots, m$ temosi $z_{i}^{a_{i}} \overline{z_{i}} b_{i}=z_{i}^{c} \|\left. w_{i}\right|^{2 b_{i}}=\left(\|\left.\left.\right|_{i}\right|^{\frac{z_{i}}{c_{i}}} z_{i}\right)^{c_{i}}=w_{i}^{c_{i}}$. Portanto o teorema.

A continuidade deste estuclo para as demais famílias estudadas om [RSV] está sendo feita por Seade o Pichon em [PS]. (min preparay̧ăo. 
() projeto para estudar a topologia de fibraça de Milnor de singularidades reais é um projeto interessante o pode levar à caracterizaçào de uma classe de singularidades cuja topologia esteja relacionada, de alguma forma, ao caso holomorfo. Este é um problema que pretendo estudar na continuidade deste trabalho.

Os resultados obtidos no capílulo 4 podem ser úteis para associar invariantes das variedardes $M_{\theta}$ à topologia de $\psi$, que poderiam distinguir as fibraçós que são topologicamente equivalentes d̀s fibraçoes holomorfits.

Uma outra direção para futıros estudos. é o caso de singularidades năo isoladas. A obrenção de condição suficiente para a frivialiclade topológica de famílias, neste caso, é um problema difícil e inmportante na teria de Singularidades [Ja2], [Ga1]. 


\section{Referências Bibliográficas}

[BL] J. Bochnak and S. Lojasiewice, A Converse of the Kuiper=Kuo Theorem, Jecture Notes in Mathematics 192, Springer, Berlin. 1971, 254261.

[BK] K. Bekka and S. Koike, The Kuo Condition, an Inequality of Thom's Type and (c)-Regularity, Topology, vol.37, 1, pp.45-62, 1998.

[C] N. A'Campo, Le Nombre de Lefschets d'une Monodromie, Indag. Math., 35, 1973. 113118.

[CA] C. Bivià-Ausina, Singularidades de. Thom-Boardman en Deformaciones Genéricas de Germes de Aplicaciones y Métodos para el Cálculo de Clausuras Integrales de Ideales, Tese de doutorado, Universitat de Valoncia, 2000.

[CR] C. R. L. Fornandes, Fecho Integral de Ideais e Whitney-Equisingularidades: Dissertação de Mestrado apresentada ao IC.MC-LSP-São Carlos, 2001.

[DG] J. Damon, e T. Gaffney, Topological Triviality of Deformations of Functions and. Newton Filtrations, Inv. Math.72, pp.335-358, 1983.

[FP] T. Fukui and Laurentiu Paunescu, Stratificalion Theory from the Weighted Point of Vieu, Canadian Journal of Mathematics, 53(2001), 73-97.

[Ga] T. Gaffiney, The Integral Closure of Modules and Whitney equisingularity, Inv. Math.102, pp.301\%322, 1992.

[Ga1] Г. Gaffiney, Polar Multiplicities and Equisingularity of Map Germs, Topology Vol. 32, No. 1, pp. $185223,1993$. 
[GR] G.-Martin Greuel, Constant Minor Number Implies Constant Multiplicity For Quasihomogeneous Singularities, Manuscripta Math.56, pp.159 166, 1986.

[HK] H. C. King, Topological Type in Families of Germs, Inventiones Matematicae, 62 , pp.1-13, 1980.

[HK1] H. King, Topological Type of Isolated Critical Points, Annals of Mathematics, 107 , 1978,385-397.

[.Ja $\mathrm{J}_{\mathrm{j}}$ A. Jacquemard, Fibrations de Milnor Pour des Applications Réelles, Boll. Un. Mat. Ital., vol.37, 1. pp. $4562,3-B, 1989$.

[Ja1] A. Jacquemard, Thèse 3ène cycle Université de Dijon, 1982.

[Ja2] A. Jacquemard, On the Fiber of the Compound of a Real Analytic Function by a Projection, Boll. Un. Mat. Ital., 8. 2-B. pp.263-278, 3-B.1999.

[.JD] J. Damon, Topological Trivality of Versal Unfoldings of Complete Intersections, Annales Inst. Fourier 34 (4)(1984).pp.225-251.

[K] T.-C. Kuo, On $\mathcal{C}^{0}$-Sufficiency of Jcts of Potential Functions, Topology, 8, 1969, 167.171 .

[Ku] N. I1. Kuiper, $C^{\prime}$-Equivalence of Functions Near Isolated Critical Points, Proc. Symp. in Infinite Dimnnsional Topology (Baton Rouge, 1967), Annals of Mathematics Studies 69 (Princeton, 1968), 199218.

[KB] K. Bekka, Regular Quasi-Homogeneous Stratifications, "Stratification, singularities and diferential equations II Stratifications and Topology of Singular Space" Travaux en cours 55, Hermann, 1997, 114.

[KB1] K. Bekka, (c)-Regularité et Trivialilé Topologique, Singularity theory and its applications, Warwick 1989, Jart I, D.Mond and J. Montaldi, Eds., SLNM 1462 , Springer, (1991), pp.42-62.

[KII] A. G. Khovanskii. Newton Polyhedra and Toroidal Varieties, Funct. Anal. and Appl.11 (1977),pp.289-295.

[Ma1] M. J. Saia, The Integral Closure of Ideals and Whitney E'quisingularity of Germs of Hypersurfaces, Matemática Compenporânea. S. B3.M. "4th Workshop on Real and Complex Singularities", Ed. M. A. S. R.uas, S. Carlos - CSP, Brasil. Vol. 12. 183-198, (1997). 
[Mi] J. Milnor, Singular Points of Complex Hypersurfaces, Ann. of Math. Studies 61, Princeton Univ. Press, 1968.

[Ma] M. J. Saia, The Integral Closure of Ideals and the Neuton Filtration, Algebraic Geometry 5, 1-11, 1996.

[NR] R. Nonato c M. A. S. Ruas, Real Milnor Fibrations and (c)-Regularity, será submetido para uma revista especializada.

[PS] A. Pichon and Seade, Real Singularities and Open-book Decomposition of the 3-sphere, em preparação.

[R] M. A. S. Ruas, On de Degree of $C^{l}-$ Determinacy, Math. Scand.59, 1986, pp.59 70.

[RS] M. A. S. Ruas e M. J. Saia, $C^{l}$-Determinacy of Weighted Homogeneous Germs, Hokkaido Math. Journal XXVI, no.1, 1997, pp.89-99.

[RSV] M. A. S. Ruas, J.Scade and Verjovsky On Real Singularities with a Milnor Fibration, Trends in Mathematics: trends in Singularities, (c) 2002 Birkhauser Verlag Basel/Switzerland, pp.191 213

[S1] J. Seade, Fibred Links and a Construction of Real Singularities vio Complex Geometry, Topology, vol.37, 1, pp.45 62, 1998.

[S2] J. Seade, Open Book Decomposition Associated to Holomorphic Vector Fields, Bol. Soc. Mat. Mexicana(3), vol.3, pp.323-336, 1997.

[Te] B. Teissier, Introduction to Equisingularity Problems, AMS Proc. Symp. Pure Maths 29 , pp.539-632, 1975.

[Ve] A. Verona, Stratified Mappings-structures and Triangulability, Lecture Notes in Math.1102, Springer Verlag(1983).

[W] C. T. C. Wall, Finite Determinacy of Smooth Map-germs, Bull. London. Math. Soc.13, pp.481-539, 1981. 